\title{
MONODROMY OF THE $p$-RANK STRATA OF THE MODULI SPACE OF CURVES
}

\author{
JEFFREY D. ACHTER, RACHEL PRIES
}

\begin{abstract}
AвSTRACT. We determine the $\mathbb{Z} / \ell$-monodromy and $\mathbb{Z}_{\ell}$-monodromy of every irreducible component of the stratum $\mathcal{M}_{g}^{f}$ of curves of genus $g$ and $p$-rank $f$ in characteristic $p$. In particular, we prove that the $\mathbb{Z} / \ell$ monodromy of every component of $\mathcal{M}_{g}^{f}$ is the symplectic group $\mathrm{Sp}_{2 g}(\mathbb{Z} / \ell)$ if $g \geq 3$ and if $\ell$ is a prime distinct from $p$. The method involves results on the intersection of $\overline{\mathcal{M}}_{g}^{f}$ with the boundary of $\overline{\mathcal{M}}_{g}$. We give applications to the generic behavior of automorphism groups, Jacobians, class groups, and zeta functions of curves of given genus and $p$-rank.
\end{abstract}

\section{INTRODUCTION}

Suppose $C$ is a smooth connected projective curve of genus $g \geq 1$ over an algebraically closed field $k$ of characteristic $p>0$. The Jacobian $\operatorname{Pic}^{0}(C)$ is a principally polarized abelian variety of dimension $g$. The number of $p$-torsion points of $\operatorname{Pic}^{0}(C)$ is $p^{f}$ for some integer $f$, called the $p$-rank of $C$, with $0 \leq f \leq g$.

Let $\mathcal{M}_{g}$ be the moduli space over $k$ of smooth connected projective curves of genus $g$; it is a smooth Deligne-Mumford stack over $k$. The $p$-rank induces a stratification $\mathcal{M}_{g}=\cup \mathcal{M}_{g}^{f}$ by locally closed reduced substacks $\mathcal{M}_{g}^{f}$, whose geometric points correspond to curves of genus $g$ and $p$-rank $f$.

Let $\ell$ be a prime number distinct from $p$. In this paper, we compute the $\ell$-adic monodromy of every irreducible component of $\mathcal{M}_{g}^{f}$. The main result implies that there is no restriction on the monodromy group other than that it preserve the symplectic pairing coming from the principal polarization. Heuristically, this means that $p$-rank constraints alone do not force the existence of extra automorphisms (or other algebraic cycles) on a family of curves.

To describe this result more precisely, let $S$ be a connected stack over $k$, and let $s$ be a geometric point of $S$. Let $C \rightarrow S$ be a relative smooth proper curve of genus $g$ over $S$. Then $\operatorname{Pic}^{0}(C)[\ell]$ is an étale cover of $S$ with geometric fiber isomorphic to $(\mathbb{Z} / \ell)^{2 g}$. The fundamental group $\pi_{1}(S, s)$ acts linearly on the fiber $\operatorname{Pic}^{0}(C)[\ell]_{s}$, and the monodromy group $\mathrm{M}_{\ell}(C \rightarrow$

Key words and phrases. monodromy, p-rank, moduli, Jacobian, curve; MSC 11G18, 11G20, $14 \mathrm{D} 05$. 
$S, s)$ is the image of $\pi_{1}(S, s)$ in $\operatorname{Aut}\left(\operatorname{Pic}^{0}(C)[\ell]_{S}\right)$. For the main result we determine $\mathrm{M}_{\ell}(S):=\mathrm{M}_{\ell}(C \rightarrow S, s)$, where $S$ is an irreducible component of $\mathcal{M}_{g}^{f}$ and $C \rightarrow S$ is the tautological curve. This also determines the $\ell$-adic monodromy group $\mathrm{M}_{\mathbb{Z}_{\ell}}(S)$.

Theorem 4.5, Let $\ell$ be a prime distinct from $p$ and suppose $g \geq 1$. Suppose $0 \leq f \leq g$, and $f \neq 0$ if $g \leq 2$. Let $S$ be an irreducible component of $\mathcal{M}_{g}^{f}$, the p-rank $f$ stratum in $\mathcal{M}_{g}$. Then $\mathrm{M}_{\ell}(S) \cong \operatorname{Sp}_{2 g}(\mathbb{Z} / \ell)$ and $\mathrm{M}_{\mathbb{Z}_{\ell}}(S) \cong \operatorname{Sp}_{2 g}\left(\mathbb{Z}_{\ell}\right)$.

We also prove an analogous result about $p$-adic monodromy (Proposition 4.8).

We give four applications of Theorem 4.5 in Section 5, The first two do not use the full strength of the theorem, in that they can be deduced solely from knowledge of the $\mathbb{Q}_{\ell}$-monodromy. Application (i) complements [26, Thm. 1] (and recovers [2, Thm. 1.1(i)]), while application (ii) complements results in [14, Thm. 1]. Applications (iii) and (iv) build upon [16, 9.7.13] and [19, 6.1] respectively.

Applications: Let $\mathbb{F}$ be a finite field of characteristic $p$. Under the hypotheses of Theorem 4.5 .

: (i) there is an $\overline{\mathbb{F}}$-curve $C$ of genus $g$ and $p$-rank $f$ with $\operatorname{Aut}_{\overline{\mathbb{F}}}(C)=$ \{id $\}$ (5.4);

: (ii) there is an $\overline{\mathbb{F}}$-curve $C$ of genus $g$ and $p$-rank $f$ whose Jacobian is absolutely simple (5.7);

: (iii) if $|\mathbb{F}| \equiv 1 \bmod \ell$, about $\ell /\left(\ell^{2}-1\right)$ of the $\mathbb{F}$-curves of genus $g$ and $p$-rank $f$ have a point of order $\ell$ on their Jacobian (5.9);

: (iv) for most $\mathbb{F}$-curves $C$ of genus $g$ and $p$-rank $f$, the splitting field of the numerator of the zeta function of $C$ has degree $2^{g} g$ ! over $\mathbb{Q}$ (5.11).

At its heart, this paper relies on fundamental work of Chai and Oort. The proof of Theorem 4.5 appears in Section 4.2. It proceeds by degeneration (as in [7]) and induction on the genus. Consider the moduli space $\mathcal{A}_{g}$ of principally polarized abelian varieties of dimension $g$ and its $p$-rank strata $\mathcal{A}_{g}^{f}$. Recent work in [5] gives information about the integral monodromy of $\mathcal{A}_{g}^{f}$. In particular, an irreducible subspace of $\mathcal{A}_{g}$ which is stable under all Hecke correspondences and whose generic point is not supersingular has monodromy group $\mathrm{Sp}_{2 g}(\mathbb{Z} / \ell)$. The base cases of Theorem 4.5 rely on the fact that the dimensions of $\mathcal{M}_{g}^{f}$ and $\mathcal{A}_{g}^{f}$ are equal if $g \leq 3$.

We note that [5] is not directly applicable to the strata $\mathcal{M}_{g}^{f}$ when $g \geq 4$. When $g \geq 4$, the Torelli locus is very far from being Hecke-stable. Another method for computing monodromy groups is found in [13], where the author shows that certain group-theoretic conditions on the local inertia structure of a $\mathbb{Z} / \ell$-sheaf guarantee that its global monodromy group is the full symplectic group. The method of [13] applies only to families of 
curves in which the Jacobian of at least one degenerate fiber has a nontrivial toric part, and thus does not apply to $\mathcal{M}_{\mathrm{g}}^{0}$.

The inductive step of Theorem 4.5 uses results about the boundary of $\mathcal{M}_{g}^{f}$ found in Section 3 . In particular, it employs a new result that the closure of every component $S$ of $\mathcal{M}_{g}^{f}$ in $\overline{\mathcal{M}}_{g}$ contains moduli points of chains of curves of specified genera and $p$-rank (Proposition 3.5), and in particular intersects the boundary component $\Delta_{1,1}$ in a certain way (Corollary 3.7). As in [3], this implies that the monodromy group of $S$ contains two non-identical copies of $\mathrm{Sp}_{2 g-2}(\mathbb{Z} / \ell)$, and is thus isomorphic to $\mathrm{Sp}_{2 g}(\mathbb{Z} / \ell)$.

A result of independent interest in Section 3 is the following.

Corollary 3.6. Suppose $g \geq 2$ and $0 \leq f \leq g$. Let $\Omega \subset\{1, \ldots, g\}$ be a subset of cardinality $f$. Let $S$ be an irreducible component of $\mathcal{M}_{g}^{f}$. Then $\bar{S}$ contains the moduli point of a chain of elliptic curves $E_{1}, \ldots, E_{g}$, where $E_{j}$ is ordinary if and only if $j \in \Omega$.

In Section 3.2, we include some open questions about the geometry of the $p$-rank strata of curves. For example, the number of irreducible components of $\mathcal{M}_{g}^{f}$ is known only in special cases. Finally, we anticipate that the techniques of this paper can be used to compute the $\ell$-adic monodromy of components of the $p$-rank strata $\mathcal{H}_{g}^{f}$ of the moduli space $\mathcal{H}_{g}$ of hyperelliptic curves of genus $g$ as well.

We thank the referee for helpful comments.

\section{BACKGROUND}

Let $k$ be an algebraically closed field of characteristic $p>0$. In Sections 2, 3, and 4 all objects are defined on the category of $k$-schemes, and $T$ is an arbitrary $k$-scheme. Let $\ell$ be a prime distinct from $p$. We fix an isomorphism $\boldsymbol{\mu}_{\ell} \simeq \mathbb{Z} / \ell$.

2.1. Moduli spaces. For each $g \geq 1$ consider the following well-known categories, each of which is fibered in groupoids over the category of $k$ schemes in its étale topology:

: $\mathcal{A}_{g}$ principally polarized abelian schemes of dimension $g$;

: $\mathcal{M}_{g}$ smooth connected proper relative curves of genus $g$;

$: \overline{\mathcal{M}}_{g}$ stable relative curves of genus $g$.

For each positive integer $r$, there is also (see [18, Def. 1.1,1.2]) the category

$: \overline{\mathcal{M}}_{g ; r} r$-labeled stable relative curves $\left(C ; P_{1}, \ldots, P_{r}\right)$ of genus $g$.

These are all smooth Deligne-Mumford stacks, and $\overline{\mathcal{M}}_{g}$ and $\overline{\mathcal{M}}_{g ; r}$ are proper [18, Thm. 2.7]. There is a forgetful functor $\phi_{g ; r}: \overline{\mathcal{M}}_{g ; r} \rightarrow \overline{\mathcal{M}}_{g}$. Let $\overline{\mathcal{M}}_{g, 0}=$ $\overline{\mathcal{M}}_{g}$. Let $\mathcal{M}_{g ; r}=\overline{\mathcal{M}}_{g ; r} \times \overline{\mathcal{M}}_{g} \mathcal{M}_{g}$ be the moduli stack of $r$-labeled smooth curves of genus $g$. The boundaries of $\overline{\mathcal{M}}_{g}$ and $\overline{\mathcal{M}}_{g ; r}$ are $\partial \overline{\mathcal{M}}_{g}=\overline{\mathcal{M}}_{g}-\mathcal{M}_{g}$ 
and $\partial \overline{\mathcal{M}}_{g ; r}=\overline{\mathcal{M}}_{g ; r}-\mathcal{M}_{g ; r}$, respectively. If $S \subset \overline{\mathcal{M}}_{g}$, let $\bar{S}$ be the closure of $S$ in $\overline{\mathcal{M}}_{g}$.

For a $k$-scheme $T, \mathcal{M}_{g}(T)=\operatorname{Mor}_{k}\left(T, \mathcal{M}_{g}\right)$ is the category of smooth proper relative curves of genus $g$ over $T$. There is a tautological curve $\mathcal{C}_{g}$ over the moduli stack $\mathcal{M}_{g}$ [6, Sec. 5]. If $s \in \mathcal{M}_{g}(k)$, let $\mathcal{C}_{g, s}$ denote the fiber of $\mathcal{C}_{g}$ over s, which is the curve corresponding to the point $s:$ Spec $k \rightarrow \mathcal{M}_{g}$. Similar conventions are employed for the tautological marked curve $\mathcal{C}_{g ; r}$ over $\overline{\mathcal{M}}_{g ; r}$.

Let $C / k$ be a stable curve. The Picard variety $\operatorname{Pic}^{0}(C)$ is an abelian variety if each irreducible component of $C$ is smooth and if the intersection graph of the irreducible components of $C$ is a tree. Such a curve is said to be of compact type. Curves which are not of compact type correspond to points of a component $\Delta_{0}$ (defined in Section 2.3) of $\partial \overline{\mathcal{M}}_{g}$.

2.2. The $p$-rank. Let $X$ be a principally polarized abelian variety of dimension $g$ over an algebraically closed field $k^{\prime}$ of characteristic $p$. The $p$-rank of $X$ is the integer $f$ such that $X[p]\left(k^{\prime}\right) \cong(\mathbb{Z} / p)^{f}$. It may be computed as $f(X)=\operatorname{dim}_{\mathbb{F}_{p}} \operatorname{Hom}\left(\boldsymbol{\mu}_{p}, X\right)$, where $\boldsymbol{\mu}_{p}$ is the kernel of Frobenius on the multiplicative group $\mathbb{G}_{m}$. It is well-known that $0 \leq f \leq g$. This definition extends to semiabelian varieties; if $X / k^{\prime}$ is a semiabelian variety, its $p$-rank is $\operatorname{dim}_{\mathbb{F}_{p}} \operatorname{Hom}\left(\boldsymbol{\mu}_{p}, X\right)$. If $X$ is an extension of an abelian variety $Y$ by a torus $W$, then $f(X)=f(Y)+\operatorname{dim}(W)$. If $X / k_{0}$ is a semiabelian variety over an arbitrary field of characteristic $p$, its $p$-rank is that of $X_{k^{\prime}}$ for any algebraically closed field $k^{\prime}$ containing $k_{0}$. If $C / k^{\prime}$ is a stable curve, then its $p$-rank $f(C)$ is that of $\operatorname{Pic}^{0}(C)$.

Lemma 2.1. Let $X \rightarrow S$ be a semiabelian scheme of relative dimension $g$ over a Deligne-Mumford stack, and suppose $0 \leq f \leq g$. There is a locally closed reduced substack $S^{f}$ of $S$ such that for each field $k^{\prime} \supset k$ and point $s \in S\left(k^{\prime}\right)$, then $s \in S^{f}\left(k^{\prime}\right)$ if and only if the $p$-rank of $X_{s}$ is $f$.

Proof. A substack of $S$ is reduced and locally closed if it is locally representable by reduced locally closed subschemes [6, p. 100] [20, 3.9 and 3.14]. Therefore, it suffices to consider the case that $S$ is an affine scheme. Write $X$ as an extension $0 \rightarrow W \rightarrow X \rightarrow Y \rightarrow 0$, where $Y$ is an abelian scheme and $W$ is a torus. Since $\operatorname{dim}(W)$ is an upper semicontinuous function on the base [10, p. 8], there is a finite stratification of $S$ by locally closed subschemes on which $\operatorname{dim}(W)$ is constant. Since a finite union of locally closed subschemes is again locally closed, one may assume that $\operatorname{dim}(W)$ is constant. Finally, since $f(Y)=f(X)+\operatorname{dim}(W)$, it suffices to prove the result for the abelian scheme $Y$. The existence of $S^{f}$ then follows immediately from [15, Thm. 3.2.1].

In particular, $\mathcal{A}_{g}^{f}$ and $\mathcal{M}_{g}^{f}$ denote the locally closed reduced substacks of $\mathcal{A}_{g}$ and $\mathcal{M}_{g}$, respectively, whose geometric points correspond to objects 
with $p$-rank $f$. Similary, $\overline{\mathcal{M}}_{g}^{f}:=\left(\overline{\mathcal{M}}_{g}\right)^{f}$ and $\overline{\mathcal{M}}_{g ; r}^{f}:=\left(\overline{\mathcal{M}}_{g: r}\right)^{f}$. Note that $\overline{\mathcal{M}}_{g}^{f}$ may be strictly contained in $\overline{\mathcal{M}_{g}^{f}}$ since the latter may contain points $s$ such that $f\left(\mathcal{C}_{g, s}\right)<f$.

Every component of $\overline{\mathcal{M}}_{g}^{f}$ has dimension $2 g-3+f$ [9, Thm. 2.3]. Since $\overline{\mathcal{M}}_{g ; r}^{f}$ is the fibre of $\phi_{g, r}$ over $\overline{\mathcal{M}}_{g ; r}^{f}$, it is pure of dimension $2 g-3+f+r$.

2.3. Clutching maps. If $g_{1}, g_{2}, r_{1}, r_{2}$ are positive integers, there is a clutching map

$$
\kappa_{g_{1} ; r_{1}, g_{2} ; r_{2}}: \overline{\mathcal{M}}_{g_{1} ; r_{1}} \times \overline{\mathcal{M}}_{g_{2} ; r_{2}} \longrightarrow \overline{\mathcal{M}}_{g_{1}+g_{2} ; r_{1}+r_{2}-2}
$$

Suppose $s_{1} \in \overline{\mathcal{M}}_{g_{1} ; r_{1}}(T)$ is the moduli point of the labeled curve $\left(C_{1} ; P_{1}, \ldots, P_{r}\right)$, and suppose $s_{2} \in \overline{\mathcal{M}}_{g_{2} ; r_{2}}(T)$ is the moduli point of $\left(C_{2} ; Q_{1}, \ldots, Q_{r_{2}}\right)$. Then $\kappa_{g_{1}, r_{1}, g_{2} ; r_{2}}\left(s_{1}, s_{2}\right)$ is the moduli point of the labeled $T$-curve $\left(D ; P_{1}, \ldots, P_{r_{1}-1}, Q_{2}, \ldots Q_{r_{2}}\right)$, where the underlying curve $D$ has components $C_{1}$ and $C_{2}$, the sections $P_{r_{1}}$ and $Q_{1}$ are identified in an ordinary double point, and this nodal section is dropped from the labeling. The clutching map is a closed immersion if $g_{1} \neq g_{2}$ or if $r_{1}+r_{2} \geq 3$, and is always a finite, unramified map [18. Cor. 3.9].

By [4, Ex. 9.2.8],

$$
\operatorname{Pic}^{0}(D) \cong \operatorname{Pic}^{0}\left(C_{1}\right) \times \operatorname{Pic}^{0}\left(C_{2}\right) .
$$

Then the $p$-rank of $E$ is

$$
f(D)=f\left(C_{1}\right)+f\left(C_{2}\right) .
$$

Similarly, if $g$ is a positive integer and if $r \geq 2$, there is a map

$$
\kappa_{g ; r}: \overline{\mathcal{M}}_{g ; r} \longrightarrow \overline{\mathcal{M}}_{g+1 ; r-2} \text {. }
$$

If $s \in \overline{\mathcal{M}}_{g ; r}(T)$ is the moduli point of the labeled curve $\left(C ; P_{1}, \ldots, P_{r}\right)$ then $\kappa_{g ; r}(s)$ is the moduli point of the labeled curve $\left(E ; P_{1}, \ldots, P_{r-2}\right)$ where $E$ is obtained by identifying the sections $P_{r-1}$ and $P_{r}$ in an ordinary double point, and these sections are subsequently dropped from the labeling. Again, the morphism $\kappa_{g ; r}$ is finite and unramified [18, Cor. 3.9].

By [4, Ex. 9.2.8], $\operatorname{Pic}^{0}(E)$ is an extension

$$
0 \longrightarrow W \longrightarrow \mathrm{Pic}^{0}(E) \longrightarrow \operatorname{Pic}^{0}(C) \longrightarrow 0,
$$

where $W$ is a one-dimensional torus. In particular, the toric rank of $\operatorname{Pic}^{0}(E)$ is one greater than that of $\operatorname{Pic}^{0}(C)$, and their maximal projective quotients are isomorphic, so that

$$
f(E)=f(C)+1
$$

For $1 \leq i \leq g-1$, let $\Delta_{i}=\Delta_{i}\left[\overline{\mathcal{M}}_{g}\right]$ be the image of $\kappa_{i, 1 ; g-i, 1}$. Note that $\Delta_{i}$ and $\Delta_{g-i}$ are the same substack of $\overline{\mathcal{M}}_{g}$. Let $\Delta_{0}=\Delta_{0}\left[\overline{\mathcal{M}}_{g}\right]$ be the 
image of $\overline{\mathcal{M}}_{g-1 ; 2}$ under $\kappa_{g-1 ; 2}$. Each $\Delta_{i}$ is an irreducible divisor in $\overline{\mathcal{M}}_{g}$, and $\partial \overline{\mathcal{M}}_{g}$ is the union of the $\Delta_{i}$ for $0 \leq i \leq\lfloor g / 2\rfloor$ (e.g., [18, p.190]). If $S$ is a stack equipped with a map $S \rightarrow \overline{\mathcal{M}}_{g}$, let $\Delta_{i}[S]$ denote $S \times \overline{\mathcal{M}}_{g} \Delta_{i}\left[\overline{\mathcal{M}}_{g}\right]$. Also define $\Delta_{i}\left[\overline{\mathcal{M}}_{g}\right]^{f}:=\left(\Delta_{i}\left[\overline{\mathcal{M}}_{g}\right]\right)^{f}$.

If $g \geq 3$, then there exists a commutative diagram of clutching maps

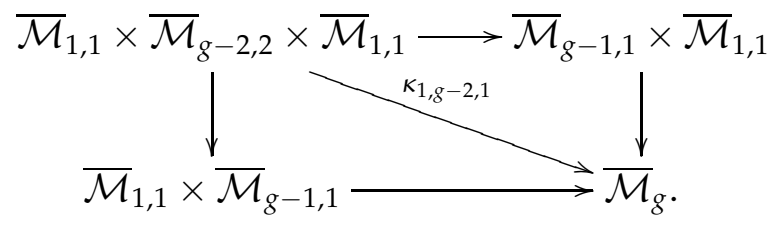

Let $\Delta_{1,1}=\Delta_{1,1}\left[\overline{\mathcal{M}}_{g}\right]$ denote the image in $\overline{\mathcal{M}}_{g}$ of the upper left-hand object; it is the (reduced) self-intersection locus of $\Delta_{1}$. There is an open, dense substack $U_{1,1} \subset \Delta_{1,1}$ such that if $s \in U_{1,1}(k)$, then $\mathcal{C}_{g, s}$ is a chain of three irreducible smooth curves $Y_{1}, Y_{2}, Y_{3}$ with $g_{Y_{1}}=g_{Y_{3}}=1$ and $g_{Y_{2}}=$ $g-2$. Also, for $i \in\{1,3\}$, the curves $Y_{i}$ and $Y_{2}$ intersect in a point $P_{i}$ which is an ordinary double point.

\section{THE $p$-RANK STRATA OF CURVES}

3.1. Boundary of the $p$-rank strata of curves. The $p$-rank strata of the boundary of $\overline{\mathcal{M}}_{g}$ are easy to describe using the clutching maps. First, if $f \geq 1$, then $\Delta_{0}\left[\overline{\mathcal{M}}_{g}\right]^{f}$ is the image of $\overline{\mathcal{M}}_{g-1 ; 2}^{f-1}$ under $\kappa_{g-1 ; 2}$ by (2.3.4). Second, if $1 \leq i \leq g-1$ and $0 \leq f \leq g$, then (2.3.2) implies that $\Delta_{i}\left[\overline{\mathcal{M}}_{g}\right]^{f}$ is the union of the images of $\overline{\mathcal{M}}_{i ; 1}^{f_{1}} \times \overline{\mathcal{M}}_{g-i ; 1}^{f_{2}}$ under $\kappa_{i ; 1, g-i ; 1}$ as $\left(f_{1}, f_{2}\right)$ ranges over all pairs such that

$$
0 \leq f_{1} \leq i, 0 \leq f_{2} \leq g-i \text { and } f_{1}+f_{2}=f .
$$

Lemma 3.1. Suppose $g \geq 2$ and $0 \leq f \leq g$. If $0 \leq i \leq g-1$ and $(f, i) \neq$ $(0,0)$, then every component of $\Delta_{i}\left[\overline{\mathcal{M}}_{g}\right]^{f}$ has dimension $2 g+f-4$.

Proof. Suppose $1 \leq f \leq g$. Then $\overline{\mathcal{M}}_{g-1 ; 2}^{f-1}$ is pure of dimension $\operatorname{dim}\left(\overline{\mathcal{M}}_{g-1}^{f-1}\right)+$ $2=2 g+f-4$. Since $\kappa_{g-1 ; 2}$ is finite, $\Delta_{0}\left[\overline{\mathcal{M}}_{g}\right]^{f}$ is pure of dimension $2 g+$ $f-4$ as well.

Similarly, suppose $0 \leq f \leq g$ and $1 \leq i \leq g-1$. Let $\left(f_{1}, f_{2}\right)$ be any pair of integers satisfying (3.1.1). Then $\overline{\mathcal{M}}_{i ; 1}^{f_{1}} \times \overline{\mathcal{M}}_{g-i ; 1}^{f_{2}}$ is pure of dimension $\operatorname{dim}\left(\overline{\mathcal{M}}_{i ; 1}^{f_{1}}\right)+\operatorname{dim}\left(\overline{\mathcal{M}}_{g-i ; 1}^{f_{2}}\right)=2 g+f-4$. Since $\kappa_{i ; 1, g-i ; 1}$ is finite, $\Delta_{i}\left[\overline{\mathcal{M}}_{g}\right]^{f}$ is pure of dimension $2 g+f-4$ as well.

The first part of the next lemma shows that if $\eta$ is a generic point of $\overline{\mathcal{M}}_{g}^{f}$, then the curve $\mathcal{C}_{g, \eta}$ is smooth. Thus no component of $\overline{\mathcal{M}}_{g}^{f}$ is contained in the boundary $\partial \overline{\mathcal{M}}_{g}$. The last part shows that one can adjust the labeling of 
an $r$-labeled curve of genus $g$ and $p$-rank $f$ without leaving the irreducible component of $\overline{\mathcal{M}}_{g ; r}^{f}$ to which its moduli point belongs.

Lemma 3.2. Suppose $g \geq 1,0 \leq f \leq g$, and $r \geq 1$.

(a) Then $\mathcal{M}_{g}^{f}$ is open and dense in $\overline{\mathcal{M}}_{g}^{f}$.

(b) Then $\mathcal{M}_{g ; r}^{f}$ is open and dense in $\overline{\mathcal{M}}_{g ; r}^{f}$.

(c) Let $S$ be an irreducible component of $\overline{\mathcal{M}}_{g ; r}^{f}$. Then $S=\phi_{g ; r}^{-1}\left(\phi_{g ; r}(S)\right)$. Equivalently, if $T$ is a $k$-scheme, if $\left(C ; P_{1}, \ldots, P_{r}\right) \in S(T)$, and if $\left(Q_{1}, \ldots, Q_{r}\right)$ is any other labeling of $C$, then $\left(C ; Q_{1}, \ldots, Q_{r}\right) \in S(T)$.

Proof. Part (a) is well-known if $g=1$. For $g \geq 2$, the result follows immediately from Lemma 3.1, since $\overline{\mathcal{M}}_{g}^{f}$ is pure of dimension $2 g+f-3[9$. Thm. 2.3]. Part (b) follows from the fact that the $p$-rank of a labeled curve depends only on the underlying curve, so that $\overline{\mathcal{M}}_{g ; r}^{f}=\overline{\mathcal{M}}_{g ; r} \times \overline{\mathcal{M}}_{g} \overline{\mathcal{M}}_{g}^{f}$.

For part (c), let $S$ be an irreducible component of $\overline{\mathcal{M}}_{g ; r}^{f}$. It suffices to show that $\phi_{g ; r}^{-1}\left(\phi_{g ; r}(S)\right) \subseteq S$. By part (b), $U=S \cap \mathcal{M}_{g ; r}$ is open and dense in $S$. Therefore, $S$ is the largest irreducible substack of $\overline{\mathcal{M}}_{g ; r}^{f}$ which contains $U$. The fibers of $\left.\phi_{g ; r}\right|_{U}$ are irreducible, so $\phi_{g ; r}^{-1}\left(\phi_{g ; r}(U)\right)$ is also an irreducible substack of $\overline{\mathcal{M}}_{g ; r}^{f}$ which contains $U$. Thus $\phi_{g ; r}^{-1}\left(\phi_{g ; r}(U)\right) \subset S$. This shows that $\phi_{g ; r}^{-1}\left(\phi_{g ; r}(U)\right)=U$.

To finish the proof, it suffices to show that a $T$-point of $S$ is a $T$-point of $\phi_{g ; r}^{-1}\left(\phi_{g ; r}(S)\right)$ for an arbitrary $k$-scheme $T$. To this end, let $\alpha=\left(C ; P_{1}, \ldots, P_{r}\right) \in$ $S(T)$, and let $\beta=\left(C ; Q_{1}, \ldots, Q_{r}\right) \in \overline{\mathcal{M}}_{g ; r}^{f}(T)$. Note that $\phi_{g ; r}(\beta)=\phi_{g ; r}(\alpha)$, and $\phi_{g ; r}(\alpha)$ is supported in the closure of $\phi_{g ; r}(U)$ in $\overline{\mathcal{M}}_{g}^{f}$. Because $\mathcal{M}_{g ; r}$ is dense in $\overline{\mathcal{M}}_{g ; r}$, it follows that $\beta$ is supported in the closure of $\phi_{g ; r}^{-1}\left(\phi_{g ; r}(U)\right)$ in $\overline{\mathcal{M}}_{g ; r}^{f}$, which is $S$.

Lemma 3.3. Suppose $g \geq 2$ and $0 \leq f \leq g$. Let $S$ be an irreducible component of $\mathcal{M}_{g}^{f}$.

(a) Then $\bar{S}$ intersects $\Delta_{0}\left[\overline{\mathcal{M}}_{g}\right]$ if and only if $f \geq 1$.

(b) If $f \geq 1$, then each irreducible component of $\Delta_{0}[\bar{S}]$ contains the image of a component of $\overline{\mathcal{M}}_{g-1,2}^{f-1}$ under $\kappa_{g-1 ; 2}$.

In other words, Lemma 3.3(b) states that if $f \geq 1$ and if $\eta$ is a generic point of $\Delta_{0}[\bar{S}]$, then the normalization $\widetilde{\mathcal{C}}_{g, \eta}$ of $\mathcal{C}_{g, \eta}$ is a smooth curve of genus $g-1$ and $p$-rank $f-1$. The containment in Lemma 3.3 (b) may be strict since $\Delta_{0}[\bar{S}]$ may contain points $s$ such that $\mathcal{C}_{g, s}$ has $p$-rank strictly less than $f$. 
Proof. If $f=0$, then equation (2.3.4) implies that $\bar{S}$ does not intersect $\Delta_{0}\left[\overline{\mathcal{M}}_{g}\right]$. If $f \geq 1$, then $\bar{S} \subset \overline{\mathcal{M}}_{g}$ is a complete substack of dimension greater than $2 g-3$. Let $\mathcal{N}_{g}=\overline{\mathcal{M}}_{g}-\Delta_{0}$ be the sublocus of curves of compact type; it is open in $\overline{\mathcal{M}}_{g}$. By [9, Lemma 2.4], a complete substack of $\mathcal{N}_{g}$ has dimension at most $2 g-3$. Thus, $\bar{S}$ is not contained in $\mathcal{N}_{g}$ and so $\bar{S}$ intersects $\Delta_{0}$ nontrivially. This completes part (a).

For part (b), recall that $\bar{S}$ is not contained in $\partial \overline{\mathcal{M}}_{g}$ by Lemma 3.2(a). Since $\overline{\mathcal{M}}_{g}, \bar{S}$ and $\partial \overline{\mathcal{M}}_{g}$ are proper, the intersection of $\bar{S}$ with the divisor $\Delta_{0}\left[\overline{\mathcal{M}}_{g}\right]$ has pure dimension $\operatorname{dim} \bar{S}-1$, which equals $\operatorname{dim}\left(\Delta_{0}\left[\overline{\mathcal{M}}_{g}\right]^{f}\right)$ by Lemma 3.1. Thus each irreducible component of $\bar{S} \cap \Delta_{0}\left[\overline{\mathcal{M}}_{g}\right]$ contains the image of some component of $\overline{\mathcal{M}}_{g-1 ; 2}^{f-1}$ under the finite morphism $\kappa_{g-1 ; 2}$. In particular, it contains the image of the moduli point of a smooth curve of genus $g-1$ and $p$-rank $f-1$.

The next result shows that the closure of each irreducible component of $\mathcal{M}_{g}^{f}$ intersects $\Delta_{i}\left[\overline{\mathcal{M}}_{g}\right]^{f}$ in every way possible.

Proposition 3.4. Suppose $g \geq 2$ and $0 \leq f \leq g$. Suppose $1 \leq i \leq g-1$ and $\left(f_{1}, f_{2}\right)$ is a pair satisfying the conditions in (3.1.1). Let $S$ be an irreducible component of $\mathcal{M}_{g}^{f}$.

(a) Then $\bar{S}$ intersects $\kappa_{i ; 1, g-i ; 1}\left(\overline{\mathcal{M}}_{i ; 1}^{f_{1}} \times \overline{\mathcal{M}}_{g-i ; 1}^{f_{2}}\right)$.

(b) Each irreducible component of the intersection contains the image of a component of $\overline{\mathcal{M}}_{i ; 1}^{f_{1}} \times \overline{\mathcal{M}}_{g-i ; 1}^{f_{2}}$.

Proposition 3.4(b) implies that if $\eta$ is a generic point of $\bar{S} \cap \kappa_{i ; 1, g-i ; 1}\left(\overline{\mathcal{M}}_{i ; 1}^{f_{1}} \times\right.$ $\left.\overline{\mathcal{M}}_{g-i ; 1}^{f_{2}}\right)$, then $\mathcal{C}_{g, \eta}$ is a chain of two smooth curves of respective genera $i$ and $g-i$ and respective $p$-ranks $f_{1}$ and $f_{2}$.

Proof. (a) implies (b): To see that (a) implies (b) for fixed $g, f, i$, and $\left(f_{1}, f_{2}\right)$, note that $\bar{S} \cap \Delta_{i}\left[\overline{\mathcal{M}}_{g}\right]^{f}$ has pure dimension $\operatorname{dim} \bar{S}-1=2 g+f-4$ by the same reasoning as in the proof of Lemma $3.3(\mathrm{~b})$. By Lemma 3.1 this is the same as the dimension of each component of $\kappa_{i ; 1, g-i ; 1}\left(\overline{\mathcal{M}}_{i ; 1}^{f_{1}} \times \overline{\mathcal{M}}_{g-i ; 1}^{f_{2}}\right)$.

Base cases: The proof of (a) is by induction on $g$ while holding $g-f$ fixed. The two base cases are when $f=0$ and when $g=2$. When $f=0$, then the only possibility for $\left(f_{1}, f_{2}\right)$ is $(0,0)$. By $3.3(\mathrm{a}), \bar{S}$ is contained in $\mathcal{N}_{g}=\overline{\mathcal{M}}_{g}-\Delta_{0}$. By [9. Lemma 2.5], $\bar{S}$ intersects $\Delta_{i}\left[\overline{\mathcal{M}}_{g}\right]$, and a point in the intersection must be in $\kappa_{i ; 1, g-i ; 1}\left(\overline{\mathcal{M}}_{i ; 1}^{0} \times \overline{\mathcal{M}}_{g-i ; 1}^{0}\right)$.

For the other base case, let $g=2$. The statement is true for $g=f=2$ (or more generally when $g=f$ ) because $\mathcal{M}_{g}^{g}$ is open and dense in $\overline{\mathcal{M}}_{g}$. If $f=1$, then $i=1$. Without loss of generality, $\left(f_{1}, f_{2}\right)=(1,0)$. Thus the next claim suffices to conclude the proof of the base cases. 
Claim: $\overline{\mathcal{M}}_{2}^{1}$ is irreducible and intersects $\kappa_{1 ; 1,1 ; 1}\left(\overline{\mathcal{M}}_{1 ; 1}^{1} \times \overline{\mathcal{M}}_{1 ; 1}^{0}\right)$.

To prove the claim, recall that the Torelli morphism $\mathcal{M}_{2} \rightarrow \mathcal{A}_{2}$ is an inclusion [24, Lemma 1.11]. Since $\operatorname{dim}\left(\mathcal{M}_{2}^{1}\right)=\operatorname{dim}\left(\mathcal{A}_{2}^{1}\right)$, and since $\mathcal{A}_{2}^{1}$ is irreducible (e.g., [8, Ex. 11.6]), it follows that $\mathcal{M}_{2}^{1}$ is irreducible. Consider a chain of two smooth elliptic curves, one of which is ordinary and one supersingular, intersecting in an ordinary double point. The moduli point of this curve is in $\overline{\mathcal{M}}_{2}^{1} \cap \kappa_{1 ; 1,1 ; 1}\left(\overline{\mathcal{M}}_{1 ; 1}^{1} \times \overline{\mathcal{M}}_{1 ; 1}^{0}\right)$.

Inductive step: Suppose that $g \geq 3$ and $1 \leq f \leq g$. The inductive hypothesis is that, given $1 \leq i^{\prime} \leq g-2$, given a pair $\left(f_{1}^{\prime}, f_{2}^{\prime}\right)$ such that $0 \leq f_{1}^{\prime} \leq i^{\prime}, 0 \leq f_{2}^{\prime} \leq g-1-i^{\prime}$, and $f_{1}^{\prime}+f_{2}^{\prime}=f-1$, and given an irreducible component $Z$ of $\mathcal{M}_{g-1}^{f-1}$, then $\bar{Z}$ intersects $\kappa_{i^{\prime} ; 1, g-1-i^{\prime} ; 1}\left(\overline{\mathcal{M}}_{i^{\prime} ; 1}^{f_{1}^{\prime}} \times\right.$ $\left.\overline{\mathcal{M}}_{g-1-i^{\prime} ; 1}^{f_{2}^{\prime}}\right)$; and, since (a) implies (b), that each irreducible component of the intersection contains a component of $\kappa_{i^{\prime} ; 1, g-1-i^{\prime} ; 1}\left(\overline{\mathcal{M}}_{i^{\prime} ; 1}^{f_{1}^{\prime}} \times \overline{\mathcal{M}}_{g-1-i^{\prime} ; 1}^{f^{\prime}}\right)$.

Let $S$ be an irreducible component of $\mathcal{M}_{g}^{f}$. Let $1 \leq i \leq g-1$, and let $\left(f_{1}, f_{2}\right)$ be a pair which satisfies (3.1.1). Possibly after exchanging $i$ with $g-i$ and $f_{1}$ with $f_{2}$, we suppose that $f_{1}>0$.

Case 1: Suppose $i>1$. Let $\left(g_{1}^{\prime}, g_{2}^{\prime}\right)=(i-1, g-i)$, and let $\left(f_{1}^{\prime}, f_{2}^{\prime}\right)=$ $\left(f_{1}-1, f_{2}\right)$. Note that $g_{1}^{\prime}+g_{2}^{\prime}=g-1$ and $f_{1}^{\prime}+f_{2}^{\prime}=f-1$. Also $0 \leq f_{j}^{\prime} \leq g_{j}^{\prime}$ and $g_{j}^{\prime} \geq 1$ for $j=1,2$. So $1 \leq g_{1}^{\prime} \leq g-2$. By Lemma 3.3(b), $\bar{S}$ contains the image of a component $\widetilde{Z}$ of $\overline{\mathcal{M}}_{g-1 ; 2}^{f-1}$ under $\kappa_{g-1 ; 2}$. The inductive hypothesis, applied to (the interior of) the component $\bar{Z}=\phi_{g-1 ; 2}(\widetilde{Z})$ of $\overline{\mathcal{M}}_{g-1}^{f-1}$, shows that $\bar{Z}$ intersects $\kappa_{g_{1}^{\prime} ; 1, g_{2}^{\prime} ; 1}\left(\overline{\mathcal{M}}_{g_{1}^{\prime} ; 1}^{f_{1}^{\prime}} \times \overline{\mathcal{M}}_{g_{2}^{\prime} ; 1}^{f_{2}^{\prime}}\right)$.

Let $\xi$ be a generic geometric point of the intersection. By part (b) of the inductive hypothesis, the curve $\mathcal{C}_{g-1, \xi}$ is a chain of two irreducible curves, $Y_{1}$ and $Y_{2}$, with respective genera $g_{1}^{\prime}$ and $g_{2}^{\prime}$ and $p$-ranks $f_{1}^{\prime}$ and $f_{2}^{\prime}$, intersecting in one point $P$ which is an ordinary double point. Let $P_{1}$ and $P_{2}$ be two distinct points of $Y_{1}-\{P\}$. Note that after identifying the points $P_{1}$ and $P_{2}$ on $Y_{1}$, one obtains a (singular) curve of genus $g_{1}^{\prime}+1=i$ with $p$-rank $f_{1}^{\prime}+1=f_{1}$. The moduli point $\widetilde{\xi}$ of the labeled curve $\left(\mathcal{C}_{g-1, \xi},\left\{P_{1}, P_{2}\right\}\right)$ lies in $\widetilde{Z}($ Lemma $3.2(\mathrm{c}))$. Then $\kappa_{g-1 ; 2}(\widetilde{\xi}) \in \bar{S} \cap \kappa_{i ; 1, g-i ; 1}\left(\overline{\mathcal{M}}_{i ; 1}^{f_{1}} \times \overline{\mathcal{M}}_{g-i ; 1}^{f_{2}}\right)$.

Case 2: Suppose $i=1$. Then $g-i=g-1$ and $f_{1}=1$. If $f_{2}=f-1>0$, then case (1) applies after reindexing. Therefore, it suffices to consider the remaining case $(i, g-i)=(1, g-1)$ and $\left(f_{1}, f_{2}\right)=(1,0)$. Let $S$ be an irreducible component of $\mathcal{M}_{g}^{1}$. Then $\bar{S}$ contains an irreducible component of $\kappa_{2 ; 1, g-2 ; 1}\left(\overline{\mathcal{M}}_{2 ; 1}^{1} \times \overline{\mathcal{M}}_{g-2 ; 1}^{0}\right)$ by case (1). By the claim above, and the implication (a) $\rightarrow$ (b), $\overline{\mathcal{M}}_{2}^{1}$ contains the image of an irreducible component of 
$\overline{\mathcal{M}}_{1 ; 1}^{1} \times \overline{\mathcal{M}}_{1 ; 1}^{0}$ under $\kappa_{1 ; 1,1 ; 1}$. Thus $\overline{\mathcal{M}}_{2 ; 1}^{1}$ contains the image of an irreducible component of $\overline{\mathcal{M}}_{1 ; 1}^{1} \times \overline{\mathcal{M}}_{1 ; 2}^{0}$ under $\kappa_{1 ; 1,1 ; 2}$. Therefore, $\bar{S}$ contains the image of an irreducible component of $\overline{\mathcal{M}}_{1 ; 1}^{1} \times \overline{\mathcal{M}}_{1 ; 2}^{0} \times \overline{\mathcal{M}}_{g-2 ; 1}^{0}$ under $\kappa_{1,1, g-2}$. In particular, $\bar{S}$ has nonempty intersection with $\kappa_{1 ; 1, g-1 ; 1}\left(\overline{\mathcal{M}}_{1 ; 1}^{1} \times \overline{\mathcal{M}}_{g-1 ; 1}^{0}\right)$.

The next result shows that components of $\overline{\mathcal{M}}_{g}^{f}$ deeply intersect the boundary of $\overline{\mathcal{M}}_{g}$.

Proposition 3.5. Suppose $g \geq 2$ and $0 \leq f \leq g$. Let $S$ be an irreducible component of $\mathcal{M}_{g}^{f}$. Suppose $g_{1}, \ldots, g_{m}$ are positive integers and $f_{1}, \ldots, f_{m}$ are integers such that $\sum g_{i}=g, \sum f_{i}=f$, and $0 \leq f_{i} \leq g_{i}$ for each $1 \leq i \leq m$. Then $\bar{S}$ contains the image of a component of

$$
\overline{\mathcal{M}}_{g_{1} ; 1}^{f_{1}} \times \overline{\mathcal{M}}_{g_{2} ; 2}^{f_{2}} \times \overline{\mathcal{M}}_{g_{3} ; 2}^{f_{3}} \times \cdots \times \overline{\mathcal{M}}_{g_{m-1} ; 2}^{f_{m-1}} \times \overline{\mathcal{M}}_{g_{m} ; 1}^{f_{m}}
$$

under the clutching map.

Proof. The proof is by induction on $m$, and is proved for all $g$ simultaneously. The case $m=1$ is trivial, while the case $m=2$ is Proposition 3.4 .

Now suppose the result is known for every genus $g^{\prime}$ and every partition of $g^{\prime}$ with at most $m-1$ parts. Suppose $g, f, g_{1}, \ldots, g_{m}$, and $f_{1}, \ldots, f_{m}$ satisfy the conditions of Proposition 3.5. Let $g^{\prime}=\sum_{i=1}^{m-1} g_{i}$ and $f^{\prime}=\sum_{i=1}^{m-1} f_{i}$. By Proposition 3.4, there exist components $Z^{\prime}$ of $\overline{\mathcal{M}}_{g^{\prime} ; 1}^{f^{\prime}}$ and $Z$ of $\overline{\mathcal{M}}_{g_{m} ; 1}^{f_{m}}$ such that $\bar{S}$ contains $\kappa_{g^{\prime} ; 1, g_{m} ; 1}\left(Z^{\prime} \times Z\right)$. By the inductive hypothesis, $\phi_{g^{\prime} ; 1}\left(Z^{\prime}\right)$ contains $k(Y)$ for some component $Y$ of

$$
\overline{\mathcal{M}}_{g_{1} ; 1}^{f_{1}} \times \overline{\mathcal{M}}_{g_{2} ; 2}^{f_{2}} \times \overline{\mathcal{M}}_{g_{3} ; 2}^{f_{3}} \times \cdots \times \overline{\mathcal{M}}_{g_{m-2} ; 2}^{f_{m-2}} \times \overline{\mathcal{M}}_{g_{m-1} ; 1}^{f_{m-1}} .
$$

Thus, $Z^{\prime}$ contains $\kappa(Y) \times \overline{\mathcal{M}}_{g^{\prime}} \overline{\mathcal{M}}_{g^{\prime} ; 1}$. By Lemma 3.2(c), one can choose the labeled point to be supported on the last component of $\mathcal{C}_{g^{\prime}, \kappa(Y)}$. Thus, $Z^{\prime}$ contains the image of a component $X$ of

$$
\overline{\mathcal{M}}_{g_{1} ; 1}^{f_{1}} \times \overline{\mathcal{M}}_{g_{2} ; 2}^{f_{2}} \times \overline{\mathcal{M}}_{g_{3} ; 2}^{f_{3}} \times \cdots \times \overline{\mathcal{M}}_{g_{m-2} ; 2}^{f_{m-2}} \times \overline{\mathcal{M}}_{g_{m-1} ; 2}^{f_{m-1}} .
$$

Then $\bar{S}$ contains the image of $X \times Z$.

The next corollary is not used in the rest of the paper, but is included to show that Proposition 3.5 generalizes [9, Lemma 2.5], which states that every component of $\overline{\mathcal{M}}_{g}^{0}$ contains the moduli point of a chain of supersingular elliptic curves.

Corollary 3.6. Suppose $g \geq 2$ and $0 \leq f \leq g$. Let $\Omega \subset\{1, \ldots, g\}$ be a subset of cardinality $f$. Let $S$ be an irreducible component of $\mathcal{M}_{g}^{f}$. Then $\bar{S}$ contains the moduli point of a chain of elliptic curves $E_{1}, \ldots, E_{g}$, where $E_{j}$ is ordinary if and only if $j \in \Omega$. 
Proof. This follows immediately from Proposition 3.5 ,

The next result is the form of Proposition 3.5 used to prove Theorem 4.5, which relies on degeneration to $\Delta_{1,1}$. We label the four possibilities for $\left(f_{1}, f_{2}, f_{3}\right)$ such that $f_{1}+f_{2}+f_{3}=f$ and $f_{1}, f_{3} \in\{0,1\}$ as follows: (A) $(1, f-2,1)$; (B) $(0, f-1,1)$; (B') $(1, f-1,0)$; and $(C)(0, f, 0)$.

Corollary 3.7. Suppose $g \geq 3$ and $0 \leq f \leq g$. Let $S$ be an irreducible component of $\mathcal{M}_{g}^{f}$.

(a) Then $\bar{S}$ intersects $\Delta_{1,1}\left[\overline{\mathcal{M}}_{g}\right]$.

(b) There is a choice of $\left(f_{1}, f_{2}, f_{3}\right)$ from cases $(A)-(C)$, and there are irreducible components $S_{1}$ of $\overline{\mathcal{M}}_{1 ; 1}^{f_{1}}$ and $S_{2}$ of $\overline{\mathcal{M}}_{g-2 ; 2}^{f_{2}}$ and $S_{3}$ of $\overline{\mathcal{M}}_{1 ; 1}^{f_{3}}$; and there are irreducible components $S_{R}$ of $\overline{\mathcal{M}}_{g-1 ; 1}^{f_{2}+f_{3}}$ and $S_{L}$ of $\overline{\mathcal{M}}_{g-1 ; 1}^{f_{1}+f_{2}}$; so that the restriction of the clutching morphisms gives a commutative diagram

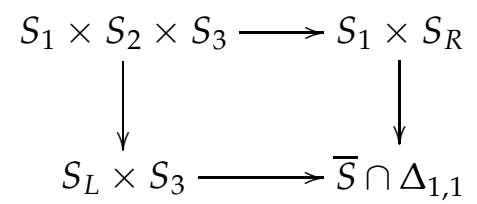

(c) Furthermore, case ( $A$ ) occurs as long as $f \geq 2$, case (B) or ( $\left.B^{\prime}\right)$ occurs as long as $1 \leq f \leq g-1$, and case $(C)$ occurs as long as $f \leq g-2$.

Proof. All three parts follow immediately from Proposition 3.5 ,

3.2. Open questions about the geometry of the $p$-rank strata. The phrasing of Corollary 3.7 is as simple as possible given the present lack of information about the number of components of $\mathcal{M}_{g}^{f}$. We note that if $\mathcal{M}_{g}^{f}$ is irreducible for some pair $g, f$, then there are much shorter proofs of Corollaries 3.6 and 3.7 .

Question 3.8. How many irreducible components does $\mathcal{M}_{g}^{f}$ have?

The answer to Question 3.8 appears to be known for all $p$ only in the following cases: $\mathcal{M}_{g}^{f}$ is irreducible when $f=g ; \mathcal{M}_{g}^{f}$ is irreducible when $g=2$ and $f \geq 1$ and when $g=3$ and $0 \leq f \leq 3$; for $\mathcal{M}_{1}^{0}$ or $\mathcal{M}_{2}^{0}$, the number of irreducible components is a class number associated with the quaternion algebra ramified at $p$ and $\infty$. If $g \geq 3$, then $\mathcal{A}_{g}^{f}$ is irreducible [5, Remark 4.7].

Question 3.9. Let $S$ be an irreducible component of $\mathcal{M}_{g}^{f}$. Does the closure of $S$ in $\mathcal{M}_{g}$ contain an irreducible component of $\mathcal{M}_{g}^{0}$ ?

The analogous property is true for $\mathcal{A}_{g}^{f}$ by [5, Remark 4.7]. If the answer to Question 3.9 is affirmative for some $g$, then one can reduce to the case $f=0$ in the proof of Theorem 4.5 . 


\section{MONODROMY}

4.1. Definition of monodromy. Recall the notations about monodromy found in [3, Section 3.1]. In particular, let $C \rightarrow S$ be a stable curve of genus $g$ over a connected $k$-scheme $S$. There is an open subset $S^{\circ} \subset S$ such that $\left.\operatorname{Pic}^{0}(C)\right|_{S^{\circ}}$ is an abelian scheme; in fact, $S^{\circ}=S \times \overline{\mathcal{M}}_{g}\left(\overline{\mathcal{M}}_{g}-\Delta_{0}\right)$. Assume that $S^{\circ}$ is nonempty and connected. Let $s \in S^{\circ}$ be a geometric point.

Let $\ell$ be a prime distinct from $p$. For each positive integer $n$, the fundamental group $\pi_{1}\left(S^{\circ}, s\right)$ acts linearly on $\operatorname{Pic}^{0}(C)\left[\ell^{n}\right]_{s}$ via a representation $\rho_{C \rightarrow S, s, \ell^{n}}: \pi_{1}\left(S^{\circ}, s\right) \rightarrow \operatorname{Aut}\left(\mathrm{Pic}^{0}(C)\left[\ell^{n}\right]_{s}\right)$. The $\mathbb{Z} / \ell^{n}$-monodromy of $C \rightarrow S$, written as $\mathrm{M}_{\ell^{n}}(C \rightarrow S, s)$, is the image of $\rho_{C \rightarrow S, s, \ell^{n}}$. If $S$ is equipped with a morphism $f: S \rightarrow \mathcal{M}$ to one of the moduli stacks $\mathcal{M}$ defined in Section 2.1, let $\mathrm{M}_{\ell^{n}}(S, s)$ denote $\mathrm{M}_{\ell^{n}}\left(f^{*} \mathcal{C} \rightarrow S, s\right)$, where $\mathcal{C} \rightarrow \mathcal{M}$ is the tautological curve. The isomorphism class of the abstract group $\mathrm{M}_{\ell^{n}}(S, s)$ is independent of $s$, and we denote it by $\mathrm{M}_{\ell^{n}}(S)$. Let $\mathrm{M}_{\mathbb{Z}_{\ell}}(S)=\lim _{\overleftarrow{n}} \mathrm{M}_{\ell^{n}}(S)$, and let $\mathrm{M}_{\mathbb{Q}_{\ell}}(S)$ be the Zariski closure of $\mathrm{M}_{\mathbb{Z}_{\ell}}(S)$ in $\mathrm{GL}_{2 g}\left(\mathbb{Q}_{\ell}\right)$.

A priori, $\mathrm{M}_{\ell^{n}}(S) \subset \mathrm{GL}_{2 g}\left(\mathbb{Z} / \ell^{n}\right)$. The principal polarization $\lambda$ on $\operatorname{Pic}^{0}(C)$ induces a symplectic pairing $\langle\cdot, \cdot\rangle_{\lambda}$ on the $\ell^{n}$-torsion, with values in $\boldsymbol{\mu}_{\ell^{n}}$. Therefore, there is an inclusion $\mathrm{M}_{\ell^{n}}(S) \subseteq \mathrm{GSp}_{2 g}\left(\mathbb{Z} / \ell^{n}\right)$ of the monodromy group in the group of symplectic similitudes. Moreover, since $k$ contains $\ell^{n}$ th roots of unity, $\mathrm{M}_{\ell^{n}}(S) \subseteq \mathrm{Sp}_{2 g}\left(\mathbb{Z} / \ell^{n}\right)$. Similarly, $\mathrm{M}_{\Lambda}(S) \subseteq \operatorname{Sp}_{2 g}(\Lambda)$ if $\Lambda$ is either $\mathbb{Z}_{\ell}$ or $\mathbb{Q}_{\ell}$. If the monodromy group is the full symplectic group, this has a geometric interpretation, as follows. Equip $(\mathbb{Z} / \ell)^{2 g}$ with the standard symplectic pairing $\langle\cdot, \cdot\rangle_{\text {std }}$, and let

$$
S_{[\ell]}:=\operatorname{Isom}\left(\left(\operatorname{Pic}^{0}(C / S)[\ell],\langle\cdot, \cdot\rangle_{\lambda}\right),\left((\mathbb{Z} / \ell)_{S}^{2 g},\langle\cdot, \cdot\rangle_{\text {std }}\right)\right) .
$$

For simplicity, assume that $S=S^{\circ}$, i.e., that all fibers of $C \rightarrow S$ are of compact type. There is an $\ell$ th root of unity on $S$, so $S_{[\ell]} \rightarrow S$ is an étale Galois cover, possibly disconnected, with covering group $\operatorname{Sp}_{2 g}(\mathbb{Z} / \ell)$. The cover $S_{[\ell]}$ is connected if and only if $\mathrm{M}_{\ell}(S) \cong \operatorname{Sp}_{2 g}(\mathbb{Z} / \ell)$.

Finally, since the category of étale covers of a Deligne-Mumford stack is a Galois category [22, Section 4], one can employ the same formalism to study a relative curve $C$ over a connected stack $\mathcal{S}$.

Let $h: \mathcal{T} \rightarrow \mathcal{S}$ be a morphism of connected stacks. This morphism is called a fibration [12, X.1.6] [22, Section A.4] if every choice of base points $t \in \mathcal{T}$ and $s \in \mathcal{S}$ with $h(t)=s$ induces an exact sequence of homotopy groups

$$
\pi_{1}\left(\mathcal{T}_{s}, t\right) \longrightarrow \pi_{1}(\mathcal{T}, t) \stackrel{h_{*}}{\longrightarrow} \pi_{1}(\mathcal{S}, s) \longrightarrow \pi_{0}\left(\mathcal{T}_{s}, t\right) \longrightarrow\{1\} .
$$

An arbitrary base change of a fibration is a fibration. If $h$ is a fibration and if the fiber $\mathcal{T}_{s}$ is connected, then $h_{*}$ is a surjection of fundamental groups. Therefore, if $\mathcal{C} \rightarrow \mathcal{S}$ is a relative curve, then the natural inclusion $\mathrm{M}_{\ell}\left(h^{*} \mathcal{C} \rightarrow\right.$ $\mathcal{T}, t) \rightarrow \mathrm{M}_{\ell}(\mathcal{C} \rightarrow \mathcal{S}, s)$ is an isomorphism. 
Lemma 4.1. Let $g$ and $r$ be positive integers. The morphism $\overline{\mathcal{M}}_{g ; r} \rightarrow \overline{\mathcal{M}}_{g}$ is a fibration with connected fibers.

Proof. By [18, p. 165], $\overline{\mathcal{M}}_{g ; r}$ is represented by $\mathcal{C}_{g ; r-1}$, the tautological (stable, labeled) curve over $\overline{\mathcal{M}}_{g ; r-1}$. The projection $\mathcal{C}_{g ; r-1} \rightarrow \overline{\mathcal{M}}_{g ; r-1}$ is proper, flat and surjective with connected fibers. By induction on $r, \phi_{g ; r}: \overline{\mathcal{M}}_{g ; r} \rightarrow$ $\overline{\mathcal{M}}_{g}$ is proper, flat and surjective, and $\phi_{g ; r}$ is a fibration [22, Section A.1].

Lemma 4.2. Let $g$ and $r$ be positive integers. Suppose $S \subset \overline{\mathcal{M}}_{g}$ is a connected substack and s is a geometric point of $S$. Let $S_{r}=S \times \overline{\mathcal{M}}_{g} \overline{\mathcal{M}}_{g ; r}$, and let $s_{r}$ be a lift of $s$ to $S_{r}$. Then $\mathrm{M}_{\ell}(S, s)=\mathrm{M}_{\ell}\left(S_{r}, s_{r}\right)$.

Proof. By Lemma 4.1, $\overline{\mathcal{M}}_{g ; r} \rightarrow \overline{\mathcal{M}}_{g}$ is a fibration with connected geometric fibers, and thus so is $S_{r} \rightarrow S$. The result now follows from the defining property (4.1.1) of fibrations.

4.2. Monodromy of the $p$-rank strata. This section contains the main result in the paper, Theorem 4.5, which is about the monodromy of the $p$-rank strata of the moduli space of curves. The proof proceeds by induction on the genus of $g$. The next lemma, due to Chai, will be used for the base case while Lemma 4.4 will be used for the inductive step.

Lemma 4.3. Let $\ell$ be a prime distinct from $p$. Let $U \subset \mathcal{A}_{g}$ be an irreducible substack which is stable under $\ell$-adic Hecke correspondences and which is not contained in the supersingular locus. Let $S$ be an irreducible component of $\mathcal{M}_{g} \times{ }_{\mathcal{A}_{g}}$ $U$. Suppose that $\operatorname{dim}(S)=\operatorname{dim}(U)$. Then $\mathrm{M}_{\ell}(S) \cong \operatorname{Sp}_{2 g}(\mathbb{Z} / \ell)$.

Proof. For the proof we introduce a fine moduli scheme $\mathcal{M}_{g,[N]}$, and then show that any irreducible component of the pullback of $S$ to $\mathcal{M}_{g,[N]}$ has full monodromy.

Fix an integer $N \geq 3$ relatively prime to $p \ell$ and an isomorphism $\boldsymbol{\mu}_{N} \cong$ $\mathbb{Z} / N$, and let $\mathcal{A}_{g,[N]}$ be the fine moduli scheme of principally polarized abelian schemes of relative dimension $g$ equipped with principal symplectic level-N structure [21, p. 139]. Let $\mathcal{M}_{g,[N]}=\mathcal{M}_{g} \times \mathcal{A}_{g} \mathcal{A}_{g,[N]}$. It is the fine moduli scheme of smooth proper curves of genus $g$ with principal symplectic level-N structure, and the induced Torelli map $\tau_{g,[N]}: \mathcal{M}_{g_{\text {, }[N]}} \rightarrow \mathcal{A}_{g_{\text {, }[N]}}$ is analyzed in [24].

Let $U_{[N]}=U \times_{\mathcal{A}_{g}} \mathcal{A}_{g,[N]}$. By [5, Prop. 4.4], $U_{[N]}$ is irreducible and $\mathrm{M}_{\ell}\left(U_{[N]}\right) \cong$ $\mathrm{Sp}_{2 g}(\mathbb{Z} / \ell)$. Let $T_{[N]}$ be any irreducible component of $S \times \mathcal{A}_{g} \mathcal{A}_{g,[N]}$. By [24, Lemma 1.11], there exists an open dense subset $V_{[N]} \subset T_{[N]}$ and an integer $d \in\{1,2\}$ such that $\left.\tau_{g,[N]}\right|_{V_{[N]}}$ has degree $d$. Moreover, if $g \leq 2$ then $d=1$. 
In summary:

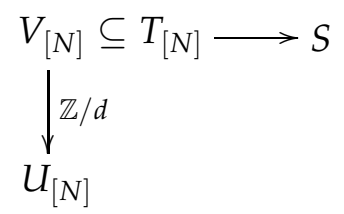

Since $\operatorname{dim}(U)=\operatorname{dim}(S), \tau_{g,[N]}\left(V_{[N]}\right)$ is dense in $U_{[N]}$. By the above conditions on $d, \operatorname{Sp}_{2 g}(\mathbb{Z} / \ell)$ has no nontrivial quotient isomorphic to $\mathbb{Z} / d$. It now follows that $\mathrm{M}_{\ell}\left(V_{[N]}\right) \cong \mathrm{Sp}_{2 g}(\mathbb{Z} / \ell)$ [3, Lemma 3.3]. Since $\mathrm{M}_{\ell}\left(V_{[N]}\right)$ is as large as possible, it follows that $\mathrm{M}_{\ell}(S) \cong \mathrm{Sp}_{2 g}(\mathbb{Z} / \ell)$.

Lemma 4.4. Let $g_{1}, g_{2}, r_{1}$ and $r_{2}$ be positive integers. Let $g=g_{1}+g_{2}$, and let $r=r_{1}+r_{2}-2$.

(a) There is a canonical isomorphism of sheaves on $\overline{\mathcal{M}}_{g_{1} ; r_{1}} \times \overline{\mathcal{M}}_{g_{2} ; r_{2}}$,

$$
\kappa_{g_{1} ; r_{1}, g_{2} ; r_{2}}^{*} \operatorname{Pic}^{0}\left(\mathcal{C}_{g ; r}\right)[\ell] \cong \operatorname{Pic}^{0}\left(\mathcal{C}_{g_{1} ; r_{1}}\right)[\ell] \times \operatorname{Pic}^{0}\left(\mathcal{C}_{g_{2} ; r_{2}}\right)[\ell] .
$$

(b) For $i=1,2$, suppose $S_{i} \subset \overline{\mathcal{M}}_{g_{i}, r_{i}}$ is connected, and let $s_{i} \in S_{i}^{\circ}$ be a geometric point. Let $S=\kappa_{g_{1} ; r_{1}, g_{2} ; r_{2}}\left(S_{1} \times S_{2}\right)$, and let $s=\kappa_{g_{1} ; r_{1}, g_{2} ; r_{2}}\left(s_{1} \times\right.$ $\left.s_{2}\right)$. Under the identification (4.2.1),

$$
\mathrm{M}_{\ell}\left(S_{1}, s_{1}\right) \times \mathrm{M}_{\ell}\left(S_{2}, s_{2}\right) \subseteq \mathrm{M}_{\ell}(S, s) \subset \operatorname{Aut}\left(\operatorname{Pic}^{0}\left(\mathcal{C}_{g ; r}\right)[\ell]_{s}\right) .
$$

Proof. The proof of [3, Lemma 3.1] applies here. The key point for part (a) is that if $C / T$ is the union of two $T$-curves $C_{1}$ and $C_{2}$, identified along a single section of $T$, then there is a canonical isomorphism $\operatorname{Pic}^{0}(C) \cong$ $\operatorname{Pic}^{0}\left(C_{1}\right) \times \operatorname{Pic}^{0}\left(C_{2}\right)(2.3 .1)$. The key ideas for part (b) involve the van Kampen theorem and covariance of the fundamental group.

Theorem 4.5. Let $\ell$ be a prime distinct from $p$ and suppose $g \geq 1$. Suppose $0 \leq f \leq g$, and $f \neq 0$ if $g \leq 2$. Let $S$ be an irreducible component of $\mathcal{M}_{g}^{f}$, the p-rank $f$ stratum in $\mathcal{M}_{g}$. Then $\mathrm{M}_{\ell}(S) \cong \operatorname{Sp}_{2 g}(\mathbb{Z} / \ell)$ and $\mathrm{M}_{\mathbb{Z}_{\ell}}(S) \cong \operatorname{Sp}_{2 g}\left(\mathbb{Z}_{\ell}\right)$.

Proof. For group-theoretic reasons (see Lemma [5.1), it suffices to show that $\mathrm{M}_{\ell}(S) \cong \mathrm{Sp}_{2 g}(\mathbb{Z} / \ell)$; for topological reasons, it suffices to show that $\mathrm{M}_{\ell}(\bar{S}) \cong \mathrm{Sp}_{2 g}(\mathbb{Z} / \ell)$.

The proof is by induction on $g$. For the base cases, suppose $1 \leq g \leq 3$ and $f \neq 0$ if $g \leq 2$. There is a Newton polygon $v_{g}^{f}$ such that the locus $U_{g}^{f}$, consisting of principally polarized abelian varieties whose Newton polygon is $v_{g}^{f}$, is open and dense in $\mathcal{A}_{g}^{f}\left[23\right.$, Thm. 2.1]. The locus $U_{g}^{f}$ is stable under Hecke correspondences. Since $f \neq 0$ if $g \leq 2, v_{g}^{f}$ is not supersingular. By [5, Rem. 4.7], $U_{g}^{f}$ is irreducible. (Alternatively, see [8, Ex. 11.6].) Let $S$ be any irreducible component of $\mathcal{M}_{g}^{f}$. Then $\operatorname{dim}(S)=\operatorname{dim}(U)$ [9. Thm. 2.3]; by Lemma4.3, $\mathrm{M}_{\ell}(S) \cong \mathrm{Sp}_{2 g}(\mathbb{Z} / \ell)$. 
Now suppose $g \geq 4$ and $0 \leq f \leq g$. As an inductive hypothesis assume, for all pairs $\left(g^{\prime}, f^{\prime}\right)$ where $g^{\prime} \geq 3$ and $0 \leq f^{\prime} \leq g^{\prime}<g$, that $\mathrm{M}_{\ell}\left(S^{\prime}\right) \cong$ $\mathrm{Sp}_{2 g^{\prime}}(\mathbb{Z} / \ell)$ for every irreducible component $S^{\prime}$ of $\mathcal{M}_{g^{\prime}}^{f^{\prime}}$.

Let $S$ be an irreducible component of $\mathcal{M}_{g}^{f}$. By Corollary $3.7, \bar{S}$ intersects $\Delta_{1,1}$ and there is a diagram as in (3.1.2). Specifically, there is a partition $f=f_{1}+f_{2}+f_{3}$, and there are irreducible components $S_{1} \subset \overline{\mathcal{M}}_{1,1}^{f_{1}}$ and $S_{2} \subset \overline{\mathcal{M}}_{g-2,2}^{f_{2}}$ and $S_{3} \subset \overline{\mathcal{M}}_{1,1}^{f_{3}}$, so that the clutching maps in (2.3.5) restrict to

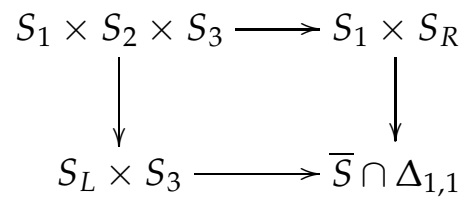

In particular, $S_{L}$ is the irreducible component of $\overline{\mathcal{M}}_{g-1,1}^{f_{1}+f_{2}}$ which contains $\kappa_{1 ; 1, g-2 ; 1}\left(S_{1} \times S_{2}\right)$, and $S_{R}$ is the irreducible component of $\overline{\mathcal{M}}_{g-1,1}^{f_{2}+f_{3}}$ which contains $\kappa_{g-2 ; 1,1 ; 1}\left(S_{2} \times S_{3}\right)$. Since $g-1 \geq 3$, the inductive hypothesis and Lemma 4.2 imply that $\mathrm{M}_{\ell}\left(S_{L}\right) \cong \mathrm{M}_{\ell}\left(S_{R}\right) \cong \mathrm{Sp}_{2(g-1)}(\mathbb{Z} / \ell)$.

Choose a base point $\left(s_{1}, s_{2}, s_{3}\right) \in\left(S_{1}^{\circ} \times S_{2}^{\circ} \times S_{3}^{\circ}\right)(k)$, and let $s=\kappa\left(s_{1}, s_{2}, s_{3}\right)$. Write $V=\operatorname{Pic}^{0}\left(\mathcal{C}_{g}\right)[\ell]_{s}$ and, for $1 \leq i \leq 3$, let $V_{i}=\operatorname{Pic}^{0}\left(\mathcal{C}_{g_{i}}\right)[\ell]_{s_{i}}$. Each of these is a $\mathbb{Z} / \ell$-vector space equipped with a symplectic form. There is an isomorphism of symplectic $\mathbb{Z} / \ell$-vector spaces $V \cong V_{1} \oplus V_{2} \oplus V_{3}$ by equation (4.2.1). By Lemma 4.4(b), $\mathrm{M}_{\ell}(S, s)$ contains $\operatorname{Sp}\left(V_{1} \oplus V_{2}\right) \times \mathrm{M}_{\ell}\left(S_{3}, s_{3}\right)$ and $\mathrm{M}_{\ell}\left(S_{1}, s_{1}\right) \times \operatorname{Sp}\left(V_{2} \oplus V_{3}\right)$. Since $\operatorname{Sp}\left(V_{1} \oplus V_{2}\right) \times \operatorname{Sp}\left(V_{3}\right)$ is a maximal subgroup of $\mathrm{Sp}(V)$ [17, Thm. 3.2], $\mathrm{M}_{\ell}(S, s) \cong \mathrm{Sp}(V) \cong \mathrm{Sp}_{2 g}(\mathbb{Z} / \ell)$.

Remark 4.6. The assertion of Theorem 4.5 is false for $\mathcal{M}_{1}^{0}$ and $\mathcal{M}_{2}^{0}$ if $\ell \geq 5$. Indeed, a curve of genus $g \leq 2$ and $p$-rank 0 is supersingular. Since a supersingular $p$-divisible group over a scheme $S$ becomes trivial after a finite pullback $\widetilde{S} \rightarrow S$, the monodromy group $\mathrm{M}_{\mathbb{Z}_{\ell}}\left(\mathcal{M}_{g}^{0}\right)$ is finite. In view of Lemma 5.1, $\mathrm{M}_{\ell}\left(\mathcal{M}_{1}^{0}\right)$ and $\mathrm{M}_{\ell}\left(\mathcal{M}_{2}^{0}\right)$ cannot be the full symplectic group.

Remark 4.7. One might wonder whether it is possible to simplify the proof of Theorem 4.5 using the intersection of $\bar{S}$ with both $\Delta_{1}$ and $\Delta_{2}$ rather than with $\Delta_{1,1}$. This does not work for the case of $\mathcal{M}_{4}^{0}$. The intersection of $\overline{\mathcal{M}}_{4}^{0}$ with $\Delta_{2}$ involves curves in $\mathcal{M}_{2}^{0}$, and the components of $\mathcal{M}_{2}^{0}$ do not have full monodromy group $\mathrm{Sp}_{4}(\mathbb{Z} / \ell)$.

4.3. The $p$-adic monodromy of the $p$-rank strata of curves. In this section we calculate the $p$-adic (as opposed to the $\ell$-adic) monodromy of components of the $p$-rank strata $\mathcal{M}_{g}^{f}$.

Let $S$ be a scheme of characteristic $p$, and let $X \rightarrow S$ be an abelian scheme with constant $p$-rank $f$. The group scheme $X[p]$ admits a largest 
étale quotient $X[p]^{\text {ét }}$ (see, e.g, [25, Cor. 1.7]). Similarly, there is a largest étale quotient $X\left[p^{\infty}\right]^{\text {ét }}$ of the $p$-divisible group $X\left[p^{\infty}\right]$. Let $s$ be a geometric point of $S$. Then $X[p]^{\text {ét }} \rightarrow S$ is classified by a homomorphism $\pi_{1}(S, s) \rightarrow$ $\operatorname{Aut}\left(X[p]^{\text {ét }}\right)_{s} \cong \mathrm{GL}_{f}(\mathbb{Z} / p)$, whose image is denoted $\mathrm{M}_{p}(X \rightarrow S)$, the physical $p$-monodromy of $X \rightarrow S$. Similarly, to $X\left[p^{\infty}\right]^{\text {ét }} \rightarrow S$ corresponds a representation $\pi_{1}(S, s) \rightarrow \operatorname{Aut}\left(X\left[p^{\infty}\right]^{\text {ét }}\right)_{s} \cong \mathrm{GL}_{f}\left(\mathbb{Z}_{p}\right)$, whose image is denoted $\mathrm{M}_{\mathbb{Z}_{p}}(X \rightarrow S)$.

Proposition 4.8. Suppose $g \geq 2$ and $1 \leq f \leq g$. Let $S$ be an irreducible component of $\mathcal{M}_{g}^{f}$. Then $\mathrm{M}_{p}(S) \cong \mathrm{GL}_{f}(\mathbb{Z} / p)$ and $\mathrm{M}_{\mathbb{Z}_{p}}(S) \cong \mathrm{GL}_{f}\left(\mathbb{Z}_{p}\right)$.

Note that this statement is not a perfect analogue of Theorem 4.5, since it only analyzes the maximal étale quotient of the $p$-divisible group of the Jacobian of the tautological curve over $S$.

Proof. If $g=f$, this is [7, Thm. 2.1]. Otherwise, suppose $f<g$. By Proposition 3.4(b), there is an irreducible component $T \subset \mathcal{M}_{g-f ; 1}^{0}$ such that $\bar{S}$ contains the image $Z$ of $\mathcal{M}_{f ; 1}^{f} \times T$ under $\kappa_{f ; 1, g-f ; 1}$. Since $M_{p}(Z)$ is isomorphic to $\mathrm{GL}_{f}(\mathbb{Z} / p)$, which is as large as possible, $\mathrm{M}_{p}(\bar{S}) \cong \mathrm{M}_{p}(S) \cong$ $\mathrm{GL}_{f}(\mathbb{Z} / p)$. Similarly, $\mathrm{M}_{\mathbb{Z}_{p}}(S) \cong \mathrm{M}_{\mathbb{Z}_{p}}(Z) \cong \mathrm{GL}_{f}\left(\mathbb{Z}_{p}\right)$.

Remark 4.9. In [29], the author proves the analogue of Proposition 4.8 for the $p$-rank strata $\mathcal{A}_{g}^{f}$. From this, he deduces the irreducibility of a certain Igusa variety, and is able to analyze the structure of the moduli space of abelian varieties with specified parahoric level- $p$ structure. The analogous statements (e.g., [29, Thm. 4.1]) hold for the moduli space of curves with parahoric level- $p$ structure, provided one replaces $\mathcal{A}_{g}^{f}$ with an irreducible component of $\mathcal{M}_{g}^{f}$.

\section{ARITHMETIC APPLICATIONS}

The results of the previous section about the monodromy of components of the moduli space of curves of genus $g$ and $p$-rank $f$ have arithmetic applications involving curves over finite fields. Specifically, they imply the existence of curves of a given type with trivial automorphism group (Application 5.4) or absolutely simple Jacobian (Application 5.7). Moreover, they give estimates for the proportion of such curves with a rational point of order $\ell$ on the Jacobian (Application 5.9) or for which the numerator of the zeta function has large splitting field (Application 5.11).

For these applications, it is necessary to work over a finite field $\mathbb{F}$, as opposed to its algebraic closure. Throughout this section, let $\mathbb{F}$ be a finite field of characteristic $p$ and cardinality $q$, and let $\overline{\mathbb{F}}$ be an algebraic closure of $\mathbb{F}$. Let $\ell$ be a prime distinct from $p$.

In this section we redefine $\mathcal{M}_{g}$ as the Deligne-Mumford stack of smooth projective curves of genus $g$ fibered over the category of $\mathbb{F}_{p}$-schemes. 
Section 5.1 contains some results on arithmetic monodromy groups, and recalls a rigidifying structure which allows one to pass between moduli stacks and moduli schemes. In Sections 5.2 and 5.3, we apply these techniques to deduce consequences for curves over finite fields.

5.1. Arithmetic monodromy and tricanonical structures. Let $\wedge$ be either $\mathbb{Z}_{\ell}, \mathbb{Q}_{\ell}$ or $\mathbb{Z} / \ell^{n}$ for some positive integer $n$. If $\pi: C \rightarrow S / \mathbb{F}$ is a smooth connected proper relative curve, its geometric $\Lambda$-monodromy group is $\mathrm{M}_{\Lambda}^{\text {geom }}(C \rightarrow$ $S)=\mathrm{M}_{\Lambda}\left(C_{\overline{\mathbb{F}}} \rightarrow S_{\bar{F}}\right)$. It is naturally a subgroup of the (arithmetic) monodromy group $\mathrm{M}_{\Lambda}(S)$. If the fibers of $\pi$ have genus $g$ one has $\mathrm{M}_{\Lambda}(S) \subseteq$ $\mathrm{GSp}_{2 g}(\Lambda)$, and

$$
\mathrm{M}_{\mathbb{Z}_{\ell}}(S) / \mathrm{M}_{\mathbb{Z}_{\ell}}^{\text {geom }}(S) \cong \operatorname{Gal}\left(\mathbb{F}\left(\boldsymbol{\mu}_{\ell \infty}(\overline{\mathbb{F}})\right) / \mathbb{F}\right) .
$$

Lemma 5.1. Let $C \rightarrow S / \mathbb{F}$ be a smooth connected proper relative curve of genus $g \geq 2$ over a geometrically connected base. If $\mathrm{M}_{\ell}^{\text {geom }}(S) \cong \operatorname{Sp}_{2 g}(\mathbb{Z} / \ell)$, then $\mathrm{M}_{\mathbb{Z}_{\ell}}^{\text {geom }}(S) \cong \mathrm{Sp}_{2 g}\left(\mathbb{Z}_{\ell}\right) ; \mathrm{M}_{\mathbb{Z}_{\ell}}(S)$ has finite index in $\mathrm{GSp}_{2 g}\left(\mathbb{Z}_{\ell}\right) ;$ and $\mathrm{M}_{\mathbb{Q}_{\ell}}^{\text {geom }}(S) \cong$ $\operatorname{Sp}_{2 g}\left(\mathbb{Q}_{\ell}\right)$.

Proof. Since $\mathrm{M}_{\mathbb{Z}_{\ell}}^{\text {geom }}(S)$ is a closed subgroup of $\mathrm{Sp}_{2 g}\left(\mathbb{Z}_{\ell}\right)$, and since $\mathrm{M}_{\mathbb{Z}_{\ell}}^{\text {geom }}(S) \hookrightarrow$ $\mathrm{Sp}_{2 g}\left(\mathbb{Z}_{\ell}\right) \rightarrow \mathrm{Sp}_{2 g}(\mathbb{Z} / \ell)$ is surjective, it follows that $\mathrm{M}_{\mathbb{Z}_{\ell}}^{\text {geom }}(S) \cong \mathrm{Sp}_{2 g}\left(\mathbb{Z}_{\ell}\right)$ for group-theoretic reasons [28, Thm. 1.3]. The remainder of the lemma follows from equation (5.1.1) and the definition of $\mathrm{M}_{\mathbb{Q}_{\ell}}$.

In Sections 5.2 and 5.3, we use Chebotarev arguments to deduce various applications about curves over finite fields. At present these tools are only available for families of curves over schemes, as opposed to stacks. To surmount this, we consider rigidifying data whose corresponding moduli problems are representable by schemes. By choosing the data $\mathcal{M}_{g, 3 \mathrm{~K}}$ of a tricanonical structure which exists Zariski-locally on the base, as opposed to a Jacobi level structure which only exists étale-locally on the base, we can relate point counts on $\mathcal{M}_{g, 3 K}(\mathbb{F})$ to those on $\mathcal{M}_{g}(\mathbb{F})$.

Suppose $g \geq 2$. The canonical bundle $\Omega_{C / S}$ is ample, and $\Omega_{C / S}^{\otimes 3}$ is very ample. Let $N(g)=5 g-5$. Then $\pi_{*}\left(\Omega_{C / S}^{\otimes 3}\right)$ is a locally free $\mathcal{O}_{S}$-module of rank $N(g)$, and sections of this bundle define a closed embedding $C \hookrightarrow$ $\mathbb{P}_{S}^{N(g)}$. A tricanonical (3K) structure on $C \rightarrow S$ is a choice of isomorphism $\mathcal{O}_{S}^{\oplus N(g)} \cong \pi_{*}\left(\Omega_{C / S}^{\otimes 3}\right)$; let $\mathcal{M}_{g, 3 K}$ be the moduli space of smooth curves of genus $g$ equipped with a $3 K$-structure. A curve with $3 K$-structure admits no nontrivial automorphisms, and $\mathcal{M}_{g, 3 K}$ is representable by a scheme [16. 10.6.5], [21, Prop. 5.1]. Moreover, $\mathcal{M}_{g}$ may be constructed as the quotient of $\mathcal{M}_{g, 3 K}$ by $\mathrm{GL}_{N(g)}$, so that the forgetful functor $\psi_{g}: \mathcal{M}_{g, 3 K} \rightarrow \mathcal{M}_{g}$ is open [21, p. 6] and a fibration [22, Thm. A.12].

Lemma 5.2. Let $S \subset \mathcal{M}_{g}$ be a connected substack, and let $S_{3 K}=S \times \mathcal{M}_{g} \mathcal{M}_{g, 3 K}$. Then $\mathrm{M}_{\ell}\left(S_{3 K}\right) \cong \mathrm{M}_{\ell}(S)$. 
Proof. Since $\mathcal{M}_{g, 3 K} \rightarrow \mathcal{M}_{g}$ is a fibration, so is $S_{3 K} \rightarrow S$. Fix a base point $s_{3 K} \in S_{3 K}(\overline{\mathbb{F}})$, and let $s=\psi_{g}\left(s_{3 K}\right)$. The fiber $S_{3 K, s}$ is connected; by the exact sequence (4.1.1), the induced homomorphism $\psi_{g *}: \pi_{1}\left(S_{3 K}, s_{3 K}\right) \rightarrow \pi_{1}(S, s)$ is surjective. As in Lemma 4.2, this implies $\mathrm{M}_{\ell}\left(S_{3 K}\right) \cong \mathrm{M}_{\ell}(S)$.

5.2. Existence applications: trivial automorphism group and simple Jacobian. In this section, we show there exist curves of genus $g$ and $p$-rank $f$ with trivial automorphism group and absolutely simple Jacobian using the $\mathbb{Q}_{\ell}$-monodromy of $\mathcal{M}_{g}^{f}$.

Lemma 5.3. Let $S \subset \mathcal{M}_{g}$ be a geometrically connected substack such that $\mathrm{M}_{\ell}^{\text {geom }}(S) \cong$ $\mathrm{Sp}_{2 g}(\mathbb{Z} / \ell)$ for all $\ell$ in a set of density one. Then there exists $s \in S(\overline{\mathbb{F}})$ such that $\operatorname{Aut}_{\overline{\mathbb{F}}}\left(\mathcal{C}_{g, s}\right)$ is either trivial or is generated by a hyperelliptic involution.

Proof. Let $S_{3 K}=S \times_{\mathcal{M}_{g}} \mathcal{M}_{g, 3 K} ;$ by Lemmas 5.1 and 5.2. $\mathrm{M}_{\mathbb{Q}_{\ell}}^{\text {geom }}\left(S_{3 K}\right) \cong$ $\mathrm{Sp}_{2 g}\left(\mathbb{Q}_{\ell}\right)$.

Let $\ell$ be a prime which splits completely in all cyclotomic fields whose degree over $\mathbb{Q}$ is at most $2 g$. By the Chebotarev density theorem (see [5. Cor. 4.3] for details), there exists an $s_{3 K} \in S_{3 K}(\overline{\mathbb{F}})$ such that $\operatorname{End}_{\overline{\mathbb{F}}}\left(\operatorname{Pic}^{0}(\mathcal{C})_{s_{3 K}}\right) \otimes$ $\mathbb{Q} \cong L$, where $L$ is a number field of dimension $[L: \mathbb{Q}]=2 g$ which is inert at $\ell$.

Since any automorphism of $\mathcal{C}_{s_{3 K}, \overline{\mathbb{F}}}$ has finite order, $\operatorname{Aut}_{\overline{\mathbb{F}}}\left(\mathcal{C}_{s_{3 K}, \overline{\mathbb{F}}}\right)$ is contained in the torsion subgroup of $\mathcal{O}_{L}^{\times}$. Since $L$ is linearly disjoint over $\mathbb{Q}$ from each cyclotomic field of degree at most $2 g$, the torsion subgroup of $\mathcal{O}_{L}^{\times}$is simply $\{ \pm 1\}$. Now, -1 has no nontrivial fixed points on the Tate module $T_{\ell}\left(\operatorname{Pic}^{0}\left(\mathcal{C}_{s_{3 K}}\right)\right)$. Therefore, if an automorphism $\iota \in \operatorname{Aut}\left(\mathcal{C}_{s_{3 K}}\right)$ acts as -1 on the Jacobian of $\mathcal{C}_{s_{3 K}}$, then the quotient of $\mathcal{C}_{S_{3 K}}$ by $\iota$ has genus zero, and $\iota$ is a hyperelliptic involution. For $s:=\psi_{g}\left(s_{3 K}\right) \in S(\overline{\mathbb{F}})$, the group $\operatorname{Aut}_{\overline{\mathbb{F}}}\left(\mathcal{C}_{g, s}\right)$ is either trivial or is generated by a hyperelliptic involution as well.

Application 5.4. Suppose $g \geq 3$ and $0 \leq f \leq g$. Then there exists an open dense substack $U \subset \mathcal{M}_{g}^{f}$ such that for each $s \in U(\overline{\mathbb{F}})$, $\operatorname{Aut}_{\overline{\mathbb{F}}}\left(\mathcal{C}_{g, s}\right)$ is trivial.

Proof. After a finite extension of the base field, one can assume that each irreducible component of $\mathcal{M}_{g}^{f}$ is geometrically irreducible. Let $S$ be one such component and recall that $\operatorname{dim}(S)=2 g-3+f$ [9, Thm. 2.3]. By Theorem 4.5, $\mathrm{M}_{\ell}^{\text {geom }}(S) \cong S \mathrm{p}_{2 g}(\mathbb{Z} / \ell)$ for all $\ell \neq p$. By Lemma 5.3, there is a nonempty (and thus open dense) substack $U_{S}^{\prime} \subset S$ whose points correspond to curves of genus $g$ and $p$-rank $f$ whose automorphism group is either trivial or generated by a hyperelliptic involution. Every component of the $p$-rank stratum $\mathcal{H}_{g}^{f}$ of the hyperelliptic locus has dimension $g-1+f$ by [11, Thm. 1] for $p \geq 3$ and by [27, Cor. 1.3] for $p=2$. Thus the intersection of $U_{S}^{\prime}$ with the hyperelliptic locus is a proper closed substack of $U_{S}^{\prime}$. 
Let $U_{S}=U_{S}^{\prime}-\left(U_{S}^{\prime} \cap \mathcal{H}_{g}^{f}\right)$, and let $U$ be the union of the stacks $U_{S}$ over all irreducible components $S$; if $s \in U(\overline{\mathbb{F}})$, then $\operatorname{Aut}_{\overline{\mathbb{F}}}\left(\mathcal{C}_{g, s}\right)$ is trivial.

Remark 5.5. Application 5.4 can be proved for $\mathcal{M}_{g}^{f}$ for all $g \geq 3$ and all $0 \leq f \leq g$ without monodromy techniques; see [2, Thm. 1.1].

Remark 5.6. In special cases, there are results in the literature that have stronger information about the field of definition of a curve with small automorphism group. In [26], the author shows that for every $p$ and every $g \geq 3$, there is a curve of genus $g$ defined over $\mathbb{F}_{p}$ with trivial automorphism group. The $p$-ranks of these curves are not determined.

Application 5.7. Suppose $g \geq 3$ and $0 \leq f \leq g$. Let $S$ be an irreducible component of $\mathcal{M}_{g}^{f}$. Then there exists $s \in S(\overline{\mathbb{F}})$ such that the Jacobian of $\mathcal{C}_{s}$ is absolutely simple.

Proof. Possibly after a finite extension of $\mathbb{F}$, one can assume that $S$ is geometrically irreducible. By Theorem 4.5, $\mathrm{M}_{\ell}^{\text {geom }}(S) \cong \mathrm{Sp}_{2 g}(\mathbb{Z} / \ell)$ for all $\ell \neq p$. By the proof of Lemma 5.3 , there exists a point $s \in S(\overline{\mathbb{F}})$ such that $\operatorname{End}_{\bar{F}}\left(\operatorname{Pic}^{0}\left(\mathcal{C}_{g, s}\right)\right) \otimes \mathbb{Q}$ is a field. Then the Jacobian $\operatorname{Pic}^{0}\left(\mathcal{C}_{g, s}\right)$ is absolutely simple.

Remark 5.8. In special cases, there are results in the literature that have stronger information about the field of definition of curves with absolutely simple Jacobians. For example, in [14] the authors show that, for every prime $p$, if $g=2$ or $g=3$, then there exists a curve with genus $g$ and $p$-rank $g$ defined over $\mathbb{F}_{p}$ whose Jacobian is absolutely simple.

5.3. Enumerative applications: class groups and zeta functions. This section contains enumerative results about curves of genus $g$ and $p$-rank $f$ that rely on $\mathbb{Z} / \ell$-monodromy groups. Recall that if $s \in \mathcal{M}_{g}(\mathbb{F})$, then $\operatorname{Pic}^{0}\left(\mathcal{C}_{g, s}\right)(\mathbb{F})$ is isomorphic to the class group of the function field $\mathbb{F}\left(\mathcal{C}_{g, s}\right)$. The size of the class group is divisible by $\ell$ exactly when there is a point of order $\ell$ on the Jacobian. Roughly speaking, Application 5.9 shows that among all curves over $\mathbb{F}$ of specified genus and $p$-rank, slightly more than $1 / \ell$ of them have an $\mathbb{F}$-rational point of order $\ell$ on their Jacobian.

Application 5.9. Suppose $g \geq 3$, and $0 \leq f \leq g$, and $\ell$ is a prime distinct from $p$. Let $\xi$ be the image of $|\mathbb{F}|$ in $(\mathbb{Z} / \ell)^{\times}$. There exists a rational function $\alpha_{g, \xi}(T) \in$ $\mathbb{Q}(T)$ such that the following holds: there exists a constant $B=B\left(\mathcal{M}_{g}^{f}, \ell\right)$ such that if $\mathcal{M}_{g}^{f}(\mathbb{F}) \neq \emptyset$, then

$$
\left|\frac{\#\left\{s \in \mathcal{M}_{g}^{f}(\mathbb{F}): \ell \text { divides }\left|\operatorname{Pic}^{0}\left(\mathcal{C}_{g, s}\right)(\mathbb{F})\right|\right\}}{\# \mathcal{M}_{g}^{f}(\mathbb{F})}-\alpha_{g, \xi}(\ell)\right|<\frac{B}{\sqrt{q}} .
$$


Remark 5.10. Suppose $\ell$ is odd. One knows that $\alpha_{g, 1}(\ell)=\frac{\ell}{\ell^{2}-1}+\mathcal{O}\left(1 / \ell^{3}\right)$, while $\alpha_{g, \xi}(\ell)=\frac{1}{\ell-1}+\mathcal{O}\left(1 / \ell^{3}\right)$ if $\xi \neq 1$. A formula for $\alpha_{g, 1}(\ell)$ is given in [1].

Proof. [Proof of 5.9] Write $\mathcal{M}$ for $\mathcal{M}_{g}^{f}$. Let $\mathcal{M}^{\text {sm }}$ be the open dense locus where the reduced stack $\mathcal{M}$ is smooth, and let $\mathcal{N}$ be the union of all connected components $S$ of $\mathcal{M}^{\text {sm }}$ such that $S(\mathbb{F}) \neq \emptyset$. Let $S$ be any such component. Since $S(\mathbb{F}) \neq \emptyset, S$ is geometrically connected and smooth, and thus geometrically irreducible. In particular, $S_{\bar{F}}$ is dense in an irreducible component of $\mathcal{M}_{\overline{\mathbb{F}}}$, and thus $\mathrm{M}_{\ell^{\prime}}^{\text {geom }}(S) \cong \mathrm{Sp}_{2 g}\left(\mathbb{Z} / \ell^{\prime}\right)$ for all $\ell^{\prime} \neq p$.

Let $S_{3 K}=S \times \mathcal{M}_{g} \mathcal{M}_{g, 3 K}$. Since the map $\psi_{g}: \mathcal{M}_{g, 3 K} \rightarrow \mathcal{M}_{g}$ is a fibration with connected fibers, $S_{3 K}$ is also connected. Tricanonical structures exist Zariski-locally, so $S_{3 K}(\mathbb{F}) \neq \emptyset$. Finally, $\psi_{g}$ is formally smooth. Taken together, this shows that $S_{3 K}$ is geometrically irreducible and smooth.

By Lemma 5.3, there is an open dense subscheme $U_{3 K}$ of $S_{3 K}$ such that if $t \in U_{3 K}$, then $\operatorname{Aut}\left(\mathcal{C}_{g, t}\right) \cong\{1\}$. The geometric monodromy group of $U_{3 K}$ is again $S_{2 g}(\mathbb{Z} / \ell$ ) (Lemma 5.2). An equidistribution theorem ([16, 9.7.13]; see also [1, 3.1]) shows that an estimate of the form (5.3.1) holds (with error term of order $\mathcal{O}(1 / \sqrt{q})$ ), where $\mathcal{M}$ is replaced by $U_{3 K}$. Let $U=\psi_{g}\left(U_{3 K}\right)$; since $\psi_{g}$ is universally open, $U$ is open, too. The fiber over each $s \in U(\mathbb{F})$ consists of exactly $\left.\left|\mathrm{GL}_{N(g)}(\mathbb{F})\right| / \mid \operatorname{Aut}\left(\mathcal{C}_{g, s}\right)\right)|=| \mathrm{GL}_{N(g)}(\mathbb{F}) \mid$ points [16, 10.6.8], and if $\psi_{g}(t)=s$ then $\mathcal{C}_{g, t} \cong \mathcal{C}_{g, s}$. Therefore, the proportion of elements $s \in U(\mathbb{F})$ for which $\ell$ divides $\left|\operatorname{Pic}^{0}\left(\mathcal{C}_{g, s}\right)(\mathbb{F})\right|$ is exactly the same as the analogous proportion of elements $t \in U_{3 K}(\mathbb{F})$.

Thus (5.3.1) holds when $\mathcal{M}$ is replaced by $U$. For dimension reasons, there exists a constant $D$ such that $\#(S-U)(\mathbb{F}) / \# S(\mathbb{F})<D / q$; therefore, (5.3.1) holds for $S$. By invoking this argument for each of the finitely many irreducible components of $\mathcal{N}$, and remembering that by construction $\mathcal{N}(\mathbb{F})=$ $\mathcal{M}^{\mathrm{sm}}(\mathbb{F})$, one obtains $(5.3 .1)$ for $\mathcal{M}^{\mathrm{sm}}(\mathbb{F})$. Finally, since there exists a constant $D^{\prime}$ such that $\#\left(\mathcal{M}-\mathcal{M}^{\mathrm{sm}}\right)(\mathbb{F}) / \# \mathcal{M}(\mathbb{F})<D^{\prime} / q$, this yields (5.3.1).

If $C / \mathbb{F}$ is a smooth projective curve of genus $g$, its zeta function has the form $P_{C / \mathbb{F}}(T) /(1-T)(1-q T)$, where $P_{C / \mathbb{F}}(T) \in \mathbb{Z}[T]$ is a polynomial of degree $2 g$. The principal polarization on the Jacobian of $C$ forces a symmetry among the roots of $P_{C / \mathbb{F}}(T)$; the largest possible Galois group for the splitting field over $\mathbb{Q}$ of $P_{C / \mathbb{F}}(T)$ is the Weyl group of $S_{2 g}$ which is a group of size $2^{g} g$ !.

Application 5.11. Suppose $g \geq 3$, and $0 \leq f \leq g$, and $p>2 g+1$. There exists a constant $\gamma=\gamma(g)>0$ so that the following holds. There exists a constant $E=E\left(\mathcal{M}_{g}^{f}\right)$ so that if $\mathcal{M}_{g}^{f}(\mathbb{F}) \neq \emptyset$, then

$\frac{\#\left\{s \in \mathcal{M}_{g}^{f}(\mathbb{F}): P_{\mathcal{C}_{g, s} / \mathbb{F}}(T) \text { is reducible, or has splitting field with degree }<2 g g !\right\}}{\# \mathcal{M}_{g}^{f}(\mathbb{F})}<E q^{-\gamma}$. 
Proof. The proof is similar to that of Application 5.9. Again, write $\mathcal{M}$ for $\mathcal{M}_{g}^{f}$. let $S$ be an irreducible component of $\mathcal{M}^{\text {sm }}$ with $S(\mathbb{F}) \neq \emptyset$, and let $U_{3 K}$ be the open dense subscheme of $S_{3 K}$ whose points correspond to curves of the specified type with tricanonical structure with trivial automorphism group. By Lemma 5.2, and Theorem 4.5, $\mathrm{M}_{\ell}^{\text {geom }}\left(U_{3 K}\right) \cong \mathrm{Sp}_{2 g}(\mathbb{Z} / \ell)$ if $\ell \neq p$. By [19, Thm. 6.1 and Remark 3.2.(4)], there is a constant $E(S)$ so that (5.3.2) is valid for $U_{3 K}$. The argument used in Application 5.9 shows the same result for each $S$, and thus for $\mathcal{M}$.

The second author was partially supported by NSF grant DMS-07-01303.

\section{REFERENCES}

[1] J. Achter. The distribution of class groups of function fields. J. Pure Appl. Algebra, 204(2):316-333, 2006.

[2] J. Achter, D. Glass, and R. Pries. Curves of given $p$-rank with trivial automorphism group. Mich. Math. J., 2008. to appear.

[3] J. Achter and R. Pries. The integral monodromy of hyperelliptic and trielliptic curves. Math. Ann., 338(1):187-206, 2007.

[4] S. Bosch, W. Lütkebohmert, and M. Raynaud. Néron models, volume 21 of Ergebnisse der Mathematik und ihrer Grenzgebiete (3) [Results in Mathematics and Related Areas (3)]. Springer-Verlag, Berlin, 1990.

[5] C.-L. Chai. Monodromy of Hecke-invariant subvarieties. Pure Appl. Math. Q., 1(2):291303, 2005.

[6] P. Deligne and D. Mumford. The irreducibility of the space of curves of given genus. Inst. Hautes Études Sci. Publ. Math., (36):75-109, 1969.

[7] T. Ekedahl. The action of monodromy on torsion points of Jacobians. In Arithmetic algebraic geometry (Texel, 1989), pages 41-49. Birkhäuser Boston, Boston, MA, 1991.

[8] T. Ekedahl and G. van der Geer. Cycle Classes of the EO Stratification on the Moduli of Abelian Varieties. In Y. Tschinkel and Y. Zarhin, editors, Algebra, arithmetic and geometry - Manin Festschrift, Progress in Mathematics. Birkhäuser, 2008. to appear.

[9] C. Faber and G. van der Geer. Complete subvarieties of moduli spaces and the Prym map. J. Reine Angew. Math., 573:117-137, 2004.

[10] G. Faltings and C.-L. Chai. Degeneration of abelian varieties. Springer-Verlag, Berlin, 1990. With an appendix by David Mumford.

[11] D. Glass and R. Pries. Hyperelliptic curves with prescribed p-torsion. Manuscripta Math., 117(3):299-317, 2005.

[12] A. Grothendieck. Revêtements étales et groupe fondamental. Springer-Verlag, Berlin, 1971. Séminaire de Géométrie Algébrique du Bois Marie 1960-1961 (SGA 1), Dirigé par Alexandre Grothendieck. Augmenté de deux exposés de M. Raynaud, Lecture Notes in Mathematics, Vol. 224.

[13] C. Hall. Big symplectic or orthogonal monodromy modulo $\ell$. Duke Math. J., 141(1):179203, 2008.

[14] E. Howe and H. J. Zhu. On the existence of absolutely simple abelian varieties of a given dimension over an arbitrary field. J. Number Theory, 92(1):139-163, 2002.

[15] N. Katz. Slope filtration of F-crystals. In Journées de Géométrie Algébrique de Rennes (Rennes, 1978), Vol. I, pages 113-163. Soc. Math. France, Paris, 1979.

[16] N. Katz and P. Sarnak. Random matrices, Frobenius eigenvalues, and monodromy. American Mathematical Society, Providence, RI, 1999.

[17] O. King. Maximal subgroups of the classical groups associated with nonisotropic subspaces of a vector space. J. Algebra, 73(2):350-375, 1981. 
[18] F. Knudsen. The projectivity of the moduli space of stable curves. II. The stacks $M_{g, n}$. Math. Scand., 52(2):161-199, 1983.

[19] E. Kowalski. The large sieve, monodromy and zeta functions of curves. J. Reine Angew. Math., 601:29-69, 2006.

[20] G. Laumon and L. Moret-Bailly. Champs algébriques, volume 39 of Ergebnisse der Mathematik und ihrer Grenzgebiete. 3. Folge. A Series of Modern Surveys in Mathematics [Results in Mathematics and Related Areas. 3rd Series. A Series of Modern Surveys in Mathematics]. Springer-Verlag, Berlin, 2000.

[21] D. Mumford, J. Fogarty, and F. Kirwan. Geometric invariant theory, volume 34 of Ergebnisse der Mathematik und ihrer Grenzgebiete (2) [Results in Mathematics and Related Areas (2)]. Springer-Verlag, Berlin, 1994.

[22] B. Noohi. Fundamental groups of algebraic stacks. J. Inst. Math. Jussieu, 3(1):69-103, 2004.

[23] F. Oort. Newton polygon strata in the moduli space of abelian varieties. In Moduli of abelian varieties (Texel Island, 1999), pages 417-440. Birkhäuser, Basel, 2001.

[24] F. Oort and J. Steenbrink. The local Torelli problem for algebraic curves. In Journées de Géometrie Algébrique d'Angers, Juillet 1979/Algebraic Geometry, Angers, 1979, pages 157204. Sijthoff \& Noordhoff, Alphen aan den Rijn, 1980.

[25] F. Oort and T. Zink. Families of $p$-divisible groups with constant Newton polygon. Doc. Math., 7:183-201 (electronic), 2002.

[26] B. Poonen. Varieties without extra automorphisms. I. Curves. Math. Res. Lett., 7(1):6776, 2000.

[27] R. Pries and H. J. Zhu. The p-rank stratification of Artin-Schreier curves. math.NT/0609657.

[28] A. Vasiu. Surjectivity criteria for p-adic representations. I. Manuscripta Math., 112(3):325-355, 2003.

[29] C.-F. Yu. Irreducibility and p-adic monodromies on the Siegel moduli spaces. 2008. arXiv:math/0612839.

Department of Mathematics, Colorado State University, Fort Collins, CO 80523 